\title{
The zebrafish as a model system for analyzing mammalian and native $\alpha$-crystallin promoter function
}

Mason Posner Corresp., ${ }^{1}$, Kelly Murray ${ }^{1}$, Matthew S McDonald ${ }^{1}$, Hayden Eighinger ${ }^{1}$, Brandon Andrew ${ }^{1}$, Am Drossman $^{1}$, Zachary Haley ${ }^{1}$, Justin Nussbaum ${ }^{2}$, Larry L David ${ }^{3}$, Kirsten J Lampi ${ }^{4}$

1 Department of Biology/Toxicology, Ashland University, Ashland, Ohio, United States of America

2 Department of Biology, Lakeland Community College, Kirtland, Ohio, United States

3 Department of Biochemistry and Molecular Biology, Oregon Health and Science University, Portland, Oregon, United States

4 Department of Integrative Biosciences, Oregon Health and Science University, Portland, Oregon, United States

Corresponding Author: Mason Posner

Email address: mposner@ashland.edu

Previous studies have used the zebrafish to investigate the biology of lens crystallin proteins and their roles in development and disease. However, little is known about zebrafish $\alpha$-crystallin promoter function, how it compares to that of mammals, or whether mammalian $\alpha$-crystallin promoter activity can be assessed using zebrafish embryos. We injected a variety of $\alpha$-crystallin promoter fragments from each species combined with the coding sequence for green fluorescent protein (GFP) into zebrafish zygotes to determine the resulting spatiotemporal expression patterns in the developing embryo. We also measured mRNA levels and protein abundance for all three zebrafish $\alpha$-crystallins. Our data showed that mouse and zebrafish $\alpha$ A-crystallin promoters generated similar GFP expression in the lens, but with earlier onset when using mouse promoters. Expression was also found in notochord and skeletal muscle in a smaller percentage of embryos. Mouse $\alpha B$-crystallin promoter fragments drove GFP expression primarily in zebrafish skeletal muscle, with less common expression in notochord, lens, heart and in extraocular regions of the eye. A short fragment containing only a lens-specific enhancer region increased lens and notochord GPF expression while decreasing muscle expression, suggesting that the influence of mouse promoter control regions carries over into zebrafish embryos. The two paralogous zebrafish $\alpha \mathrm{B}$-crystallin promoters produced subtly different expression profiles, with the aBa promoter driving expression equally in notochord and skeletal muscle while the $\alpha \mathrm{Bb}$ promoter resulted primarily in skeletal muscle expression. Messenger RNA for zebrafish $\alpha \mathrm{A}$ increased between 1 and $2 \mathrm{dpf}$, $\alpha \mathrm{Ba}$ increased between 4 and $5 \mathrm{dpf}$, but $\alpha \mathrm{Bb}$ remained at baseline levels through $5 \mathrm{dpf}$. Parallel reaction monitoring (PRM) mass spectrometry was used to detect $\alpha \mathrm{A}, \mathrm{aBa}$, and $\alpha \mathrm{Bb}$ peptides in digests of zebrafish embryos. In whole embryos, $\alpha \mathrm{A}$-crystallin was first detected by $2 \mathrm{dpf}$, peaked in abundance by 4-5 dpf, and was localized to the eye. $\alpha$ Ba was detected in whole embryo at 
nearly constant levels from 1-6 dpf, was also localized primarily to the eye, and its abundance in extraocular tissues decreased from 4-7 dpf. In contrast, due to its low abundance, no $\alpha \mathrm{Bb}$ protein could be detected in whole embryo, or dissected eye and extraocular tissues. Our results show that mammalian $\alpha$-crystallin promoters can be efficiently screened in zebrafish embryos and that their controlling regions are well conserved. An ontogenetic shift in zebrafish aBa-crystallin promoter activity provides an interesting system for examining the evolution and control of tissue specificity. Future studies that combine these promoter based approaches with the expanding ability to engineer the zebrafish genome via techniques such as CRISPR/Cas9 will allow the manipulation of protein expression to test hypotheses about lens crystallin function and its relation to lens biology and disease. 
1 The zebrafish as a model system for analyzing mammalian 2 and native $\alpha$-crystallin promoter function

3

4 Mason Posner ${ }^{1}$, Kelly Murray ${ }^{1}$, Matthew S. McDonald ${ }^{1}$, Hayden Eighinger ${ }^{1}$, Brandon

5 Andrew $^{1}$, Amy Drossman ${ }^{1}$, Zachary Haley ${ }^{1}$, Justin Nussbaum², Larry L. David ${ }^{3}$ and

6 Kirsten J. Lampi ${ }^{4}$

7 1Department of Biology, Ashland University, Ashland, $\mathrm{OH}, \mathrm{USA}$

8 2Department of Biology, Lakeland Community College

9 '3epartment of Biochemistry and Molecular Biology, Oregon Health and Science

10 University

$11{ }^{4}$ Department of Integrative Biosciences, Oregon Health and Science University

14

15

Corresponding author:

Mason Posner ${ }^{1}$

Email address: mposner@ashland.edu 
22

23

24

25

26

27

28

29

30

31

32

33

34

35

36

37

38

39

40

41

42

43

44

45

46

47

48

49

50

51

52

53

54

55

56

57

58

59

60

61

\section{Abstract:}

Previous studies have used the zebrafish to investigate the biology of lens crystallin proteins and their roles in development and disease. However, little is known about zebrafish $\alpha$-crystallin promoter function, how it compares to that of mammals, or whether mammalian $\alpha$-crystallin promoter activity can be assessed using zebrafish embryos. We injected a variety of $\alpha$-crystallin promoter fragments from each species combined with the coding sequence for green fluorescent protein (GFP) into zebrafish zygotes to determine the resulting spatiotemporal expression patterns in the developing embryo. We also measured mRNA levels and protein abundance for all three zebrafish $\alpha$-crystallins. Our data showed that mouse and zebrafish $\alpha A$-crystallin promoters generated similar GFP expression in the lens, but with earlier onset when using mouse promoters. Expression was also found in notochord and skeletal muscle in a small percentage of embryos. Mouse $\alpha B$-crystallin promoter fragments drove GFP expression primarily in zebrafish skeletal muscle, with less common expression in notochord, lens, heart and in extraocular regions of the eye. A short fragment containing only a lensspecific enhancer region increased lens and notochord GPF expression while decreasing muscle expression, suggesting that the influence of mouse promoter control regions carries over into zebrafish embryos. The two paralogous zebrafish $\alpha \mathrm{B}$-crystallin promoters produced subtly different expression profiles, with the $\alpha$ Ba promoter driving expression equally in notochord and skeletal muscle while the $\alpha \mathrm{Bb}$ promoter resulted primarily in skeletal muscle expression. Messenger RNA for zebrafish $\alpha$ a increased between 1 and $2 \mathrm{dpf}, \alpha \mathrm{Ba}$ increased between 4 and $5 \mathrm{dpf}$, but $\alpha \mathrm{Bb}$ remained at baseline levels through $5 \mathrm{dpf}$. Parallel reaction monitoring (PRM) mass spectrometry was used to detect $\alpha \mathrm{A}, \alpha \mathrm{Ba}$, and $\alpha \mathrm{Bb}$ peptides in digests of zebrafish embryos. In whole embryos, $\alpha$ A-crystallin was first detected by $2 \mathrm{dpf}$, peaked in abundance by $4-5$ $\mathrm{dpf}$, and was localized to the eye. $\alpha$ Ba was also detected in whole embryo at nearly constant levels from 1-6 dpf, was also localized primarily to the eye, and its abundance in extraocular tissues decreased from 4-7 dpf. In contrast, due to its low abundance, no $\alpha \mathrm{Bb}$ protein could be detected in whole embryo, or dissected eye and extraocular tissues. Our results show that mammalian $\alpha$-crystallin promoters can be efficiently screened in zebrafish embryos and that their controlling regions are well conserved. An ontogenetic shift in zebrafish $\alpha \mathrm{Ba}$-crystallin promoter activity provides an interesting system for examining the evolution and control of tissue specificity. Future studies that combine these promoter based approaches with the expanding ability to engineer the zebrafish genome via techniques such as CRISPR/Cas9 will allow the manipulation of protein expression to test hypotheses about lens crystallin function and its relation to lens biology and disease. 
62

63

64

65

66

67

68

69

70

71

72

73

74

75

76

77

78

79

80

81

82

83

84

85

86

87

88

89

90

91

92

93

94

95

96

97

98

99

100

101

102

103

104

105

106

107

Introduction:

The zebrafish has become a valuable model system for examining lens development, disease and the function of lens crystallin proteins. Multiple studies have identified genes and proteins involved in lens formation (Yang et al., 2004; Yang \& Cvekl, 2005; Vihtelic, 2008) and taken advantage of zebrafish embryo transparency to produce detailed imagery of lens development (Greiling \& Clark, 2009). Patterns of lens development are similar between zebrafish and mammals, with a prominent exception being that in mammals the lens placode invaginates into the lens vesicle, while in zebrafish lens fiber cells delaminate from the placode (Greiling, Aose \& Clark, 2010). Changes in the zebrafish lens proteome during its development have been described (Greiling, Houck \& Clark, 2009; Greiling, Aose \& Clark, 2010; Wages et al., 2013). The crystallin protein content of the zebrafish lens has been detailed (Posner, Kantorow \& Horwitz, 1999; Runkle et al., 2002; Wistow et al., 2005; Smith et al., 2006; Posner et al., 2008), and functional studies have examined zebrafish $\alpha$-crystallin chaperone-like activity and stability in comparison to mammals (Dahlman et al., 2005; Koteiche et al., 2015). Multiple ocular diseases, such as glaucoma, diabetic retinopathy, macular degeneration and cataract have been modeled in the zebrafish (Morris, 2011; Gestri, Link \& Neuhauss, 2012; Chhetri, Jacobson \& Gueven, 2014). In total these studies illustrate the benefits of using the zebrafish to study lens biology and provide insights into the normal function and dysfunction of the vertebrate lens.

One area of zebrafish lens biology that has not been well explored is the activity and function of lens crystallin promoters. Kurita et al. (2003) cloned the zebrafish $\alpha$ Acrystallin promoter region and used it to drive the expression of diphtheria toxin in the lens to study developmental connections between lens and retina. Davidson et al. (2003) used a Xenopus $\gamma$-crystallin promoter to express green fluorescent protein (GFP) in the zebrafish lens. Goishi et al. (2006) constructed a zebrafish $\alpha$ A-crystallin promoter/GFP plasmid to show how the zebrafish cloche mutant, which lacks a functional DNA-binding transcription factor implicated in vascular development (Reischauer et al., 2016), might downregulate $\alpha A$-crystallin expression. To our knowledge, no work since these studies has utilized zebrafish crystallin promoters, and no study has characterized the temporal or spatial expression of reporter genes linked to these promoters.

The function of mammalian $\alpha$-crystallin promoters has been the subject of multiple studies. Examination of the shared promoter region between $\alpha B$-crystallin and $H s p B 2$ in the mouse identified specific regions that enhance $\alpha \mathrm{B}$-crystallin expression. For example, an enhancer spanning -426/-259 was required for extralenticular expression while a more proximal region from $-164 /+44$ produced reporter gene expression in lens (Dubin et al., 1991; Gopal-Srivastava, Kays \& Piatigorsky, 2000; Swamynathan \& Piatigorsky, 2002). To recapitulate endogenous expression of mouse $\alpha \mathrm{B}$-crystallin, four kilobases of the 5'-flanking promoter sequence was needed (Haynes, Duncan \& Piatigorsky, 1996). A region spanning -111 to +46 of the mouse $\alpha A$-crystallin promoter was shown to drive expression of GFP in both cultured lens cells and in the mouse, with this expression enhanced by inclusion of a distal enhancer approximately 8 kilobases 
108 upstream of the gene (Yang \& Cvekl, 2005; Yang et al., 2006). While no published

109 studies report the use of mouse lens crystallin promoters in the zebrafish, Hou et al.

110 (2006) showed that a fragment of the human $\beta B 1$-crystallin promoter produced

111 transgenic expression of GFP in the zebrafish lens. A subsequent study used this

112 human promoter to drive the expression of novel proteins in the zebrafish lens to

113 examine the function of aquaporin water channels (Clemens et al., 2013). The

114 evolutionary conservation of lens crystallin gene regulation is not surprising considering

115 the similar expression of lens crystallin proteins between zebrafish and mammals

116 (Posner et al., 2008; Greiling, Houck \& Clark, 2009). This conservation suggests that

117 mammalian $\alpha$-crystallin promoters could be functionally assessed in the zebrafish,

118 providing a faster and less expensive system than traditional mouse transgenic

119 approaches. The growing development of zebrafish gene editing techniques would

120 greatly expand the capabilities of this system. Data on crystallin promoter activity would

121

122

123

124

125

126

127

128

129

130

131

132

133

134

135 also facilitate the expression of introduced proteins in zebrafish lens and other tissues.

A comparison of mouse and zebrafish $\alpha$-crystallin promoter activity can also help detail the evolution of tissue specific expression. Past studies have examined the evolution of crystallin gene expression at different timescales. For example, sequence comparisons have detailed the recruitment of crystallins during the initial evolution of the vertebrate lens, finding that the $\alpha$-crystallins are related to extra-lenticular small heat shock proteins (Wistow \& Piatigorsky, 1988). A subsequent gene duplication event was followed by divergence in expression between the two resulting paralogs $(\alpha A$ and $\alpha B$ crystallin). A more recent evolutionary change in the regulation of $\alpha$-crystallins was investigated in the blind mole rat, in which the $\alpha \mathrm{B}$-crystallin promoter has specifically lost lens activity, presumably reflecting the degenerated eyes of this subterranean species (Hough et al., 2002). In this present study we further examine evolutionary changes in $\alpha$-crystallin expression by comparing promoter activity of the two divergently expressed zebrafish $\alpha \mathrm{B}$-crystallin paralogs. While the expression of these proteins is already known in adults, an examination of their gene's promoter activities and protein abundance during early development can identify possible ontogenetic shifts in expression. The structure, stability, chaperone-like activity and expression pattern of zebrafish $\alpha \mathrm{Bb}$-crystallin is similar to the mouse ortholog (Dahlman et al., 2005; Smith et al., 2006). We predicted that this conservation would extend into early development. However, it is an open question whether the altered expression of the lens-specific zebrafish $\alpha \mathrm{Ba}$-crystallin begins in early development, or appears later in ontogeny.

Our results suggest that the zebrafish can be used as a time efficient and cost effective model for screening the activity of mammalian lens crystallin promoters. Comparison between orthologous mouse and zebrafish promoter activity supports the hypothesis that $\alpha$-crystallin promoter function is conserved between these species. Our comparative promoter analysis of the two zebrafish $\alpha \square$-crystallins shows a subtle difference in expression, and timing of developmental upregulation, between these two paralogs. We also show that zebrafish $\alpha$ Ba-crystallin undergoes an ontogenetic shift in its expression to become lens-specific later in development.

\section{Materials and Methods:}




\section{Zebrafish Maintenance and Breeding}

$A B$ or ZDR strain zebrafish were housed in $10 \mathrm{~L}$ aquaria on a recirculating filtering system maintained at $28-30^{\circ} \mathrm{C}$ with a $14: 10 \mathrm{~h}$ light and dark cycle. Fish were fed twice each day with either commercial flake food or live Artemia brine shrimp. Two males and two females were placed in one liter breeding tanks the afternoon prior to morning egg collections. Plastic dividers were used to separate the two sexes until eggs were needed to assure that all embryos were of similar ages. All animal procedures were approved by Ashland University's Animal Care Committee (approval number MP 20151).

\section{Comparative analysis of $\alpha$-crystallin promoter regions}

The UCSC Genome Browser (http://genome.ucsc.edul; (Kent et al., 2002) was used to identify conserved regions in the mouse and zebrafish $\alpha \mathrm{A}$ - and $\alpha \mathrm{B}$-crystallin promoters (Supplemental Fig. 1). A previous analysis of syntenic relations was used to assess the rearrangement of gene relationships after duplication of zebrafish $\alpha$ B-crystallin (Elicker \& Hutson, 2007).

\section{Promoter expression plasmid construction, embryo injection and assessment of GFP expression}

Primers used to amplify regions of each $\alpha$-crystallin promoter were designed using DNA Main Workbench based on sequences in GenBank and ordered from Sigma Genosys (Table 1). Each promoter region was then amplified from a bacterial artificial chromosome (BAC) clone obtained from the BACPAC Resources Center (bacpac.chori.org) using Platinum Pfx DNA polymerase (Thermo Fisher; Waltham MA). Amplification conditions were optimized to produce single bands of the expected size, which were then subcloned into the pJET1.2 plasmid (Thermo Fisher) and sequenced to confirm their identity (Functional Biosciences; Madison, WI). Restriction enzyme sites designed into each amplification primer were used to digest and ligate each cloned promoter into the pAcGFP1-1 plasmid (Clontech; Mountain View CA) using enzymes from New England Biolabs (Ipswich, MA). NEB 5 alpha cells (NEB) were transformed with each promotor/GFP construct. Some promoter constructs were produced using an alternate Gibson Assembly approach using the company's protocol (NEB). All cloned promoters have been deposited with Addgene. A promoter/GFP construct for mouse $\alpha A$-crystallin was provided by Dr. Ales Cvekl.

To prepare promoter expression plasmids for injection into zebrafish embryos, plasmids were linearized with Notl, purified using a QIAquick PCR Purification kit (Qiagen, Valencia, CA) and then dialyzed with TE buffer using a $0.025 \mu \mathrm{m}$ VSWP membrane (Millipore, Billerica, MA). Injection solutions contained $35 \mathrm{ng} / \mathrm{ul}$ of the dialyzed plasmids, $0.2 \%$ phenol red and a sufficient volume of $0.1 \mathrm{M} \mathrm{KCl}$ to produce 5 microliters of injection mix. Two nanoliters of this solution was injected into one-cell stage zebrafish embyros with a Harvard Apparatus PL-90 picoinjector (Holliston, MA) using needles prepared with a Sutter P97 Micropipette Puller (Novato, CA). Injection pressures were adjusted to inject $1 \mathrm{nl}$ of plasmid solution with each $20 \mathrm{~ms}$ pulse. Injected embryos and uninjected controls were incubated at $28{ }^{\circ} \mathrm{C}$ in fish system water and transferred to 0.2 
$200 \mathrm{mM}$ PTU at 24-30 hours post fertilization to block melanin production and facilitate

201

202

203

204

205

206

207

208

209

210

211

212

213

214

215

216

217

218

219

220

221

222

223

224

225

226

227

228

229

230

231

232

233

234

235

236

237

238

239

240

241

242

243

244

245 observation of GFP expression.

The presence of any GFP expression was examined using an Olympus IX71 inverted microscope and imaged with a SPOT RT3 camera (Diagnostic Instruments, Sterling Heights, MI). Live embryos were anesthetized in tricaine and imaged at 100X or 200X total magnification using UV illumination and GFP filter. Confocal images were captured on a Leica SP5 microscope after embryos were anesthetized and fixed in $4 \%$ paraformaldehyde. Embryos for confocal imaging were mounted on slides using Vectashield (Vector Laboratories, Burlingame, CA). Image series were then rendered as three-dimensional surface projections using Volocity imaging software (Perkin Elmer, Waltham, MA)

Quantitative PCR analysis of $\alpha$-crystallin expression in embryos All qPCR reactions were conducted in the investigators' laboratory and were designed to meet MIQE guidelines when possible as described below (Bustin et al., 2009). ZDR strain zebrafish were placed in breeding tanks that separated males and females until tank dividers were removed. Resulting fertilized eggs were collected and incubated in petri dishes containing system water in a $28^{\circ} \mathrm{C}$ incubator. Embryos were removed at 12 hours post fertilization (hpf), $24 \mathrm{hpf}, 2$ days post fertilization (dpf) $3 \mathrm{dpf}, 4 \mathrm{dpf}$ and $5 \mathrm{dpf}$ and chilled on ice before replacing system water with RNAlater (Thermo Fisher) and then stored in a $-20^{\circ} \mathrm{C}$ freezer until RNA purification. Embryos were stored between 1 hour and several days. Approximately 15 embryos were used from each timepoint for RNA purification, and three different sets of embryos were collected at each timepoint to produce three biological replicates. Total RNA from each sample was purified using an RNEasy Minikit (QIAGEN) with Qiashreddor and quantified with a NanoDrop 1000 Spectrophotometer (Thermo Scientific). Chorions were not removed from embryos that had not yet hatched. Purified total RNA $(2,000 \mathrm{ng})$ from each sample was treated with DNasel (NEB) and $6 \mu \mathrm{l}$ was used to synthesize cDNA using the Protoscript II First Strand cDNA Synthesis Kit (NEB) with the oligo $d(T)_{23}$ primer. The resulting cDNA sample was calculated to contain the equivalent of $16 \mathrm{ng} / \mu \mathrm{l}$ of original purified RNA.

All cDNA samples (three biological replicates for each timepoint) were amplified using Luna Universal qPCR Master Mix (NEB) on an Applied Biosystems StepOne Real-Time PCR System (Thermo Fisher). We used three endogenous control primer sets previously published in past studies (Tang et al., 2007; McCurley \& Callard, 2008) and a primer pair for each of the three zebrafish alpha crystallins as designed by Elicker \& Hutson (2007). All primers used and related information are shown in Table 2. Each reaction was performed in triplicate using cDNA equivalent to $32 \mathrm{ng}$ of initial purified RNA and each primer at a final concentration of $250 \mathrm{~nm}$ in $20 \mu$ reactions with the following parameters: hold at $95^{\circ} \mathrm{C}$ for 1 minute; 40 cycles of $95^{\circ} \mathrm{C}$ for $15 \mathrm{sec}$ and $60^{\circ} \mathrm{C}$ for 30 seconds; fast ramp setting.

Melt curve analysis was used to confirm that a single product was produced $\left(95^{\circ} \mathrm{C}\right.$ for 15 seconds, $60^{\circ} \mathrm{C}$ for one minute). A set of qPCR products from each primer pair was electrophoretically analyzed on a gel as well to confirm that products were the 
246 appropriate size. Each qPCR product was also sequenced to confirm that primers had amplified the correct gene. Water was used as a non-template control to detect the presence of any contaminating DNA. Parallel RNA samples from every timepoint and biological replicate that had not been treated with reverse transcriptase were amplified in duplicate as a -RT control.

The Applied Biosystems StepOne software (version 2.1) was used to calculate $\mathrm{Ct}$ values for each reaction using the software's default settings. All Ct values were exported and further calculations made in Excel (Supplemental spreadsheet 1). The Ct values for the three technical replicates from each endogenous control reaction were calculated. Forty-nine out of 51 sets of technical triplicates produced $\mathrm{Ct}$ values within 0.5 cycles. The averaged triplicate $\mathrm{Ct}$ values for each of the three endogenous control gene were then themselves averaged to produce an overall average for each sample. The $\mathrm{Ct}$ values produced by each of the three $\alpha$-crystallin primer pairs was also calculated for every cDNA sample by averaging the values from each technical triplicate. A delta $\mathrm{Ct}$ was then calculated for every cDNA sample for each $\alpha$-crystallin gene by subtracting the average endogenous control $\mathrm{Ct}$ for that sample from the values measured with the $\alpha$-crystallin specific primer pair. The result was a delta $\mathrm{Ct}$ for each of three biological replicates for three $\alpha$-crystallin genes at each timepoint. These values were imported into the statistical package $R$ (R Core Team)(using R Studio (RStudio Team)) and visualized by box and whisker plots. $R$ was also used to determine any statistically significant differences between timepoints for each alpha crystallin (ANOVA with post-hoc Tukey's HSD test).

Standard curves were generated for each primer pair to measure efficiency percentage. Purified RNA from adult zebrafish lenses was used as template for these standard curves as alpha crystallin expression in embryos was too low to produce amplification across a large range of template concentrations. Lenses were surgically removed from anesthetized adult zebrafish and purified total RNA was DNasel treated prior to cDNA synthesis as described above.

\section{Proteomic analysis of $\alpha$-crystallin content in zebrafish}

A pair of lenses from adult zebrafish were dissected, placed in $100 \mu \mathrm{l}$ of $50 \mathrm{mM}$ ammonium bicarbonate buffer, and probe sonicated ( $3 \times 5 \mathrm{sec}$ with cooling on ice between treatments) to produce a uniform suspension. The protein concentration was then determined using a BCA assay (Thermo Fisher) using BSA as a standard. A $50 \mu \mathrm{g}$ portion of protein was then reduced, alkylated, and trypsinized in the presence of ProteaseMax ${ }^{\mathrm{TM}}$ detergent using the manufacturer's recommended protocol (ProMega). Following digestion, trifluoroacetic acid was added at a final $0.5 \%$ concentration, the sample centrifuged at $16,000 \times \mathrm{g}$ for $5 \mathrm{~min}$, and the supernatant transferred to an autosampler vial. One $\mu \mathrm{g}$ of digest was then loaded onto an Acclaim PepMap $0.1 \times 20$ $\mathrm{mm}$ NanoViper C18 peptide trap (Thermo Fisher) for $5 \mathrm{~min}$ at a $5 \mu \mathrm{l} / \mathrm{min}$ flow rate in a $0.1 \%$ formic acid mobile phase. Peptides were then separated using a PepMap RSLC C18, $2 \mu \mathrm{m}$ particle, $75 \mu \mathrm{m} \times 25 \mathrm{~cm}$ EasySpray column (Thermo Fisher) and 7.5-30\% acetonitrile gradient over $60 \mathrm{~min}$ in mobile phase containing $0.1 \%$ formic acid at a 300 
$292 \mathrm{nl} / \mathrm{min}$ flow rate using a Dionex NCS-3500RS UltiMate RSLCnano UPLC system. Data-

293 dependent tandem mass spectrometry data was collected using an Orbitrap Fusion

294 Tribrid mass spectrometer configured with an EasySpray NanoSource (Thermo Fisher).

295 Survey scans from $400-1600 \mathrm{~m} / \mathrm{z}$ were performed in the Orbitrap mass analyzer at

296120,000 resolution, automatic gain control (AGC) setting of $4.0 \times 10^{5}, 50 \mathrm{msec}$

297

298

299

300

301

302

303

304

305

306

307

308

309

310

311

312

313

314

315 maximum injection time, and lock mass using a $\mathrm{m} / \mathrm{z}=445.12$ polysiloxane ion. Datadependent MS2 scans on peptide ions with signal intensities higher than 5,000, ranging from +2 to +6 charge state, and passing the monoisotopic precursor selection filter were selected for higher energy collision dissociation (HCD) with a 30\% collision energy using quadrupole isolation with a $1.6 \mathrm{~m} / \mathrm{z}$ window. Fragment ions were then analyzed in the linear ion trap with an AGC setting of $1.0 \times 10^{4}$, maximum injection time (MIT) of 35 msec, dynamic exclusion enabled, repeat count of 1 , exclusion duration of $30 \mathrm{sec}$, exclusion mass tolerance of $+/-10 \mathrm{ppm}$, top speed mode, and $3 \mathrm{sec}$ dwell time between Orbitrap survey scans. MS/MS results were then matched to peptide sequences using Sequest HT software within the Protein Discoverer 1.4 suite (Thermo Fisher) using a UniProt database containing the taxon identifier 7955 (Danio rerio) generated in July 2016 and containing 58,290 entries. Searches were performed with trypsin specificity, a maximum of 2 missed cleavages, precursor and fragment ion tolerances of $10 \mathrm{ppm}$ and $1 \mathrm{Da}$, respectively for parent and daughter ions using monoistopic masses. A static modification of +57.02 Da was added to all cysteine residues due to alkylation with iodoacetamide, and a variable modification of +15.99 Da for methionine oxidation. Peptide identifications were filtered with the Percolator node in Protein Discoverer using a reverse sequence database strategy to estimate peptide false discovery. The resulting Protein Discoverer .msf file was then imported into Skyline software (version 3.6.0.10162) (MacLean et al., 2010) to create a spectral library using identified peptides with Percolator q scores $\leq 0.05$, having between $8-25$ residues, and no missed cleavages. Three peptides each for entries Q8UUZ6 ( $\alpha$ A-crystallin), Q9PUR2 ( $\alpha B a-$ crystallin), and Q6DG35 ( $\alpha$ Bb-crystallin) were selected based on manual observation of parent ion intensities and quality of fragment ion spectra. These were then used to create a parallel reaction monitoring method to detect the presence of the three $\alpha$ crystallins during embryo development.

Uniform suspensions of either whole embryos or dissected embryo eyes and trunks were created using either probe sonication in $50 \mathrm{mM}$ ammonium bicarbonate as above, or by vortexing vigorously for $30 \mathrm{~min}$ in $20 \mu \mathrm{l}$ of $50 \mathrm{mM}$ ammonium bicarbonate buffer containing $0.2 \%$ ProteaseMax detergent. Following a protein assay, from 10-50 $\mu \mathrm{g}$ of each suspension was digested using the ProteaseMax protocol as recommended by the manufacturer, and $2 \mu \mathrm{g}$ of each digest analyzed by LC/MS using the same chromatographic separation and instrument as above, except using a parallel reaction monitoring method (Bourmaud, Gallien \& Domon, 2016) to detect the 9 targeted $\alpha$ crystallin peptide ions (Supplemental Table 1). Peptides were isolated and fragmented as above, except without data-dependency and by cycling through the list of ions throughout the chromatographic separation so the intensity of fragment ions could be continuously monitored. MS/MS spectra were acquired in the instrument's Orbitrap mass analyzer at a resolution of 30,000 , AGC setting of $5 \times 10^{4}, 100 \mathrm{msec}$ MIT, with a scan range of $\mathrm{m} / \mathrm{z} 200-2000$. Skyline was then used to extract intensities for the three 
338 most intense fragment ions for each peptide determined from the lens spectral library,

339 and perform peak detection and integration to monitor the relative abundance of $\alpha$ -

340 crystallins during embryo development.

341

342

343

344

345

346

347

348

349

350

351

352

353

354

355

356

357

358

359

360

361

362

363

364

365

366

367

368

369

370

371

372

373

374

375

376

377

378

379

380

381

382

383

\section{Results:}

The location of green fluorescent protein (GFP) expression resulting from injection of mouse and zebrafish promoters into zebrafish zygotes was examined by both standard fluorescent microscopy and confocal microscopy. Representative confocal images of anatomical structures that expressed GFP during this study are shown in Fig. 1. Video fly-throughs of representative structures can be found in Supplemental Videos 1-3.

Patterns and timelines of expression produced by each promoter can be found in Tables 3 and 4, and are described below. Overall 1622 observations were made of 616 individual injected embryos ranging in age from 24 hours post fertilization (hpf) to 7 days post fertilization (dpf). GFP expression was seen in $76.0 \%$ of examined embryos.

Mouse and zebrafish $\alpha A$-crystallin promoters produced similar GFP expression in zebrafish embryos with a subtle difference in timing

Previous work has shown strong conservation in $\alpha \mathrm{A}$-crystallin DNA sequences, protein stability and chaperone-like activity between zebrafish and mammals (Runkle et al., 2002; Dahlman et al., 2005; Posner et al., 2012). The zebrafish and mouse $\alpha A$ crystallin orthologs are similarly arranged relative to other genes, with both located in a head-to-head orientation with heat shock factor binding protein gene hsf2bp (Fig. 2a). However, mouse and human contain a second gene, salt inducible kinase 1 between $\alpha \mathrm{A}$ and $h s f 2 b p$, and the intergenic distances are much greater (Wolf et al., 2008). Several sequence regions of the mouse $\alpha \mathrm{A}$-crystallin promoter are conserved in the zebrafish genome (Supplemental Fig. 1a). Here we show that a mouse $\alpha A$-crystallin promoter fragment $(-111$ to +46$)$ combined with enhancer region DCR1 drove green fluorescent protein expression in the zebrafish lens, with much less common expression in skeletal muscle (Fig. 2b-c; Table 3). This pattern was similar to that produced by a 1 $\mathrm{kb}$ fragment of the zebrafish $\alpha \mathrm{A}$ promoter (Fig. $2 \mathrm{~d}-\mathrm{e}$ ). The zebrafish promoter also produced spots of GFP expression dorsal to the yolk that were much less common with the mouse promoter (Fig. 1g-h). A small fraction of embryos injected with the zebrafish and mouse $\alpha A$-crystallin promoters showed GFP expression in segments of the notochord. There was a subtle difference in onset of expression between the two promoters, with GFP driven by the mouse $\alpha A$ promoter noticeable by 30 hours post fertilization (hpf) while the zebrafish $\alpha \mathrm{A}$ promoter became active between 30 and $48 \mathrm{hpf}$ (Table 4).

Mouse $\alpha B$-crystallin promoter drove GFP expression in zebrafish embryos Previous studies have shown that an upstream enhancer region (-426/-257) of the mouse $\alpha \mathrm{B}$-crystallin promoter is required for extralenticular expression, while a more proximal region $(-164 /+44)$ is sufficient for driving lens expression (Dubin et al., 1991; Gopal-Srivastava \& Piatigorsky, 1994) (Fig. 3a). Genomic sequence alignment shows two areas of conservation between mouse and zebrafish in this proximal promoter region (Supplemental Fig. 1b). We cloned three mouse $\alpha B$-crystallin promoter 
384 fragments into a GFP plasmid to examine whether these functional regions had similar 385 effect in zebrafish. Our results indicate that the mouse $\alpha \mathrm{B}$-crystallin promoter drives 386 GFP expression in zebrafish embryo skeletal muscle, notochord, lens and heart (Fig. 3). The presence of the upstream enhancer region increased expression in skeletal muscle and heart compared to the shorter $250 \mathrm{bp}$ fragment (Table 3). The 0.8 and $1.5 \mathrm{~kb}$ promoters both produced skeletal muscle GFP expression in a large percentage of embryos (94 and 100\%), while this percentage was a lower $79 \%$ when using the $250 \mathrm{bp}$ fragment. (Table 3). Heart GFP expression was reduced from $6 \%$ and $12 \%$ to $2 \%$ with the 250 bp promoter. However, exclusion of the upstream enhancer increased the number of embryos expressing GFP in the lens and notochord (Table 3). The $250 \mathrm{bp}$ promoter fragment also led to slightly earlier GFP expression (by 24 hours post fertilization) than the longer $800 \mathrm{bp}$ and $1.5 \mathrm{~kb}$ fragments (Table 4).

\section{The two zebrafish $\alpha B$-crystallins produced different patterns of GFP expression} The presence of two $\alpha \mathrm{B}$-crystallins in the zebrafish is likely the result of an ancient genome duplication event at the base of teleost evolution (Van de Peer, Taylor \& Meyer, 2003). This duplication resulted in a divergence in chromosomal arrangement. Zebrafish $\alpha \mathrm{Ba}$ is located on chromosome 15 along with several distant genes with which its ortholog shows syntenic relationship in mammals (Elicker \& Hutson, 2007). Zebrafish $\alpha \mathrm{Bb}$ has maintained the same tail-to-tail organization with fellow heat shock protein $\mathrm{Hspb2}$ as is found in mammals, however the intergenic region in the zebrafish is much larger, at 6 kilobases compared to $1 \mathrm{~kb}$ in the mouse (Fig. 4a).

Previous studies indicated that the expression pattern of zebrafish $\alpha \mathrm{Ba}$ - and $\alpha \mathrm{Bb}-$ crystallin differs in adults, and proteomic analysis showed a difference in the timing of expression onset (Posner, Kantorow \& Horwitz, 1999; Smith et al., 2006; Wages et al., 2013). However, no study has characterized developmental patterns produced by the two respective promoter regions. We produced a GFP-linked $3 \mathrm{~kb}$ fragment of the zebrafish $\alpha \mathrm{Ba}$-crystallin promoter and a series of GFP-linked fragments spanning the expanded $\alpha \mathrm{Bb}$-crystallin promoter. We found no difference between the timing of onset for any of these zebrafish promoters, with GFP first appearing between 30 and $48 \mathrm{hpf}$ (Table 4). We also found no difference in timing or spatial expression between the $\alpha \mathrm{Bb}-$ crystallin promoter fragments, suggesting that sequences upstream of $1 \mathrm{~kb}$ do not regulate expression of this gene.

The spatial expression of GFP produced by all of the zebrafish $\alpha \mathrm{B}$-crystallin promoters was similar, with expression common in skeletal muscle and notochord (Fig. 4 b-c). However, the prevalence of GFP in these two tissues differed, with the zebrafish $\alpha \mathrm{Ba}$ promoter driving GFP equally (54.9\% in skeletal muscle and $56.3 \%$ in notochord) while zebrafish $\alpha \mathrm{Bb}$ promoters were much more active in skeletal muscle than notochord $(95.5 \%$ versus $10.3 \%$; Fig. $4 \mathrm{~d})$. Both zebrafish $\alpha \mathrm{B}$ promoters produced very rare GFP expression in lens (7 embryos out of 357 observed; Fig. 4e), some expression in the eye peripheral to the lens (22 embryos) and three $\alpha \mathrm{Bb}$ promoter-injected embryos, out of 267, produced GFP expression in the heart. Overall these data suggest that the divergent expression of the two zebrafish $\alpha \mathrm{B}$-crystallin promoters previously identified in 
429 adults appears later in development than the 1-7 dpf window examined in this present 430 study.

431

432

433

434

435

436

437

438

439

440

441

442

443

444

445

446

447

448

449

450

451

452

453

454

455

456

457

458

459

460

461

462

463

464

465

466

467

468

469

470

471

472

473

Measurement of zebrafish $\alpha$-crystallin transcription by $q P C R$

We used quantitative polymerase chain reaction (qPCR) to measure the concentration of $\alpha$-crystallin mRNA during zebrafish embryo development. Messenger RNA from all three $\alpha$-crystallins was detectable at $0.5 \mathrm{dpf}$, the earliest time point analyzed (Fig. 5). The variation in $\mathrm{Ct}$ values within each technical triplicate for this and other early timepoints was high for all three $\alpha$-crystallins, suggesting that $\alpha$-crystallin expression was very low at these early developmental stages. Transcription of $\alpha \mathrm{A}$-crystallin increased between 1 and $2 \mathrm{dpf}$, while $\alpha \mathrm{Ba}$-crystallin expression increased between 4 and $5 \mathrm{dpf}$. Variation in Ct values within each technical triplicate decreased to less than 0.5 as expression increased. Transcription levels for $\alpha \mathrm{Bb}$-crystallin remained consistently low through $5 \mathrm{dpf}$. Ct values for all three $\alpha$-crystallins were much higher than those for the three reference genes, suggesting that expression of all $\alpha$-crystallins was relatively low. All $\mathrm{Ct}$ values and delta $\mathrm{Ct}$ value calculations are shown in Supplemental Spreadsheet 1.

Proteomic analysis identified two of three $\alpha$-crystallins in zebrafish embryos We used a mass spectrometric parallel reaction monitoring approach to identify the presence of $\alpha$-crystallins in pooled zebrafish embryos at 1 to $6 \mathrm{dpf}$ as a complement to the promoter expression and qPCR data presented above. Two of the three targeted $\alpha \mathrm{A}$-crystallin peptides were detected by $2 \mathrm{dpf}$ and peaked in abundance at $4-5 \mathrm{dpf}$. The results for $\alpha A$ peptide 52-65 from whole embryo digests are shown in Fig. 6a, while Fig. $6 \mathrm{~b}$ shows the relative abundance of $\alpha \mathrm{A}$-crystallin in dissected eye and remaining trunks at 4 and $7 \mathrm{dpf}$. These results indicate that $\alpha A$-crystallin peptide was largely present only in eye. Its apparent decrease in abundance in whole embryos by 6 dpf was likely due to its dilution by non-ocular proteins during embryonic development. While only one $\alpha \mathrm{Ba}$-crystallin was detected in embryo digests, its measurement indicated that $\alpha \mathrm{Ba}-$ crystallin was present in almost equal abundance from 1-6 dpf (Fig. 6c). While small amounts of $\alpha \mathrm{Ba}$-crystallin were detected in $4 \mathrm{dpf}$ trunks, the protein was not detected in trunks by $7 \mathrm{dpf}$ (Fig. 6d). However, $\alpha \mathrm{Ba}$-crystallin was present in eye and appeared to increase from 4 to $7 \mathrm{dpf}$. The decrease in $\alpha \mathrm{Ba}$ in trunk and concurrent increase in eye is consistent with its unaltered abundance in whole embryos from 1-6 dpf. No $\alpha \mathrm{Bb}-$ crystallin peptides were detected in either whole embryos or dissected eyes or trunks. The extracted ion chromatograms from these parallel reaction monitoring experiments are shown in Supplementary Figures 2-8.

\section{Discussion:}

This present study is the first to show that mammalian $\alpha$-crystallin promoter function can be analyzed in zebrafish embryos by observing green fluorescent protein (GFP) expression, suggesting that the zebrafish can be used as an efficient model for mammalian $\alpha$-crystallin promoter analysis. We also provide the first data characterizing the activity of the three zebrafish $\alpha$-crystallin gene promoters. These data detail their 
474 spatiotemporal expression and identify differences between embryonic and adult 475 expression patterns for the duplicated and divergent zebrafish $\alpha \mathrm{B}$-crystallin paralogs.

476 Future studies can use the techniques described here to measure the expression 477 potential of modified lens crystallin promoters. Our data also show how different 478 crystallin promoters could be used to drive the expression of target genes in specific

479

480

481

482

483

484

485

486

487

488

489

490

491

492

493

494

495

496

497

498

499

500

501

502

503

504

505

506

507

508

509

510

511

512

513

514

515

516

517

518

Our data show that mouse $\alpha$-crystallin promoters successfully drive expression in zebrafish embryos. An interesting question is whether the resulting expression patterns match that expected in the mouse, or alternatively, if the zebrafish embryos read the mouse promoter in their own way. Is mouse promoter activity modified by the signaling molecule environment of the zebrafish? This question is difficult to answer for the mouse $\alpha A$-crystallin promoter as the GFP expression produced in zebrafish embryos was very similar to that of the native zebrafish ortholog. Similar expression profiles produced by each $\alpha \mathrm{A}$-crystallin promoter could be due to evolutionarily conserved roles in development, or alternatively that the zebrafish embryo reads the mouse promoter as one of its own. Interestingly, the mouse $\alpha A$ promoter expressed GFP at a slightly younger age than the native zebrafish promoter. This difference was also seen when comparing the mouse and zebrafish $\alpha \mathrm{B}$-crystallin promoters. Some element in the mouse promoter sequences appears to have accelerated the timing of expression. Earlier studies showed that the transcription factors Pax6, c-Maf, and CREB regulate expression of mouse $\alpha A$-crystallin (Yang \& Cvekl, 2005) and that FGF signaling regulates expression of c-Maf (Xie et al., 2016). We hypothesize that teleost fishes use a similar regulatory system, although the presence of two Pax6 genes in zebrafish may alter the details of this regulation (Kleinjan et al., 2008). Analysis of mouse and chicken $\beta B 1-c r y s t a l l i n$ promoter regions showed similar cross-species conservation in regulation, with some differences that indicated additional regulatory elements for lensspecific expression in the mouse promoter (Chen et al., 2001). The lack of detected $\alpha A$ peptides outside of the lens and low prevalence of extraocular GFP expression resulting from $\alpha \mathrm{A}$-promoters is consistent with the interpretation that the presence of $\alpha \mathrm{A}$-crystallin protein outside the lens is low throughout zebrafish development.

Comparison of mouse and zebrafish $\alpha \mathrm{B}$-crystallin promoter function is complicated, and potentially more interesting, because of the presence of two $\alpha \mathrm{B}$-crystallin paralogs in the zebrafish. Zebrafish $\alpha \mathrm{Ba}$-crystallin protein is largely restricted to the lens in adults while $\alpha \mathrm{Bb}$-crystallin is found ubiquitously, similar to the single mammalian ortholog (Posner, Kantorow \& Horwitz, 1999; Smith et al., 2006). Since duplicated $\alpha B$-crystallins are only known from teleost fishes such as the zebrafish, the restriction of expression to the lens likely evolved after the genome duplication event in this taxon (Van de Peer, Taylor \& Meyer, 2003). There are several interesting observations to note about the comparisons between mouse and zebrafish orthologs and between the two zebrafish paralogs. First, the mouse $\alpha \mathrm{B}$-crystallin promoter drove lens GFP expression in a larger percentage of embryos than either zebrafish $\alpha \mathrm{B}$-crystallin promoter. This result may 
519 reflect the lower overall abundance of $\alpha$-crystallin in the zebrafish lens compared to 520 mammals (Posner et al., 2008), and previously observed low levels of $\alpha \mathrm{Ba}$ - and $\alpha \mathrm{Bb}$ -

521 crystallin expression in early zebrafish development (Greiling, Houck \& Clark, 2009;

522 Wages et al., 2013). Second, the proportion of embryos expressing GFP in notochord

523 and skeletal muscle varied between the three different promoters. The zebrafish $\alpha \mathrm{Bb}$

524 promoter was a strong driver of GFP expression in skeletal muscle, like the mouse $\alpha B$

525 promoter, but was not as active in the notochord. The zebrafish $\alpha$ Ba promoter was

526 active in notochord, like the mouse promoter, but less active in skeletal muscle. These

527 observations are consistent with a hypothesis that mammalian $\alpha \mathrm{B}$-crystallin function has

528 been divided between the two zebrafish paralogs. While the function of $\alpha B$-crystallins in

529 zebrafish notochord and skeletal muscle at these developmental stages is not known, it

530 is possible that some developmental functions in these tissues have been divided

531 between the two zebrafish paralogs as well. Signaling sequences from an original

532 teleost $\alpha B$-crystallin gene have possibly become split between the two current zebrafish

533 paralogs. Finally, as mentioned above, the mouse $\alpha B$-promoter initiated GFP

534

535

536

537

538

539

540

541

542

543

544

545 expression at an earlier stage than the zebrafish orthologs.

The length of each zebrafish $\alpha \mathrm{Bb}$-crystallin promoter fragment had no noticeable effect on GFP expression, suggesting that any regulatory elements influencing this gene's activity remain within the first $1 \mathrm{~kb}$ upstream of the start codon. Regulatory elements do not appear to have been "stretched out" with the inclusion of additional sequence between zebrafish $\alpha \mathrm{Bb}$-crystallin and Hspb2. We did, however, see a noticeable difference in expression when a mouse $\alpha \mathrm{B}$-crystallin promoter without the skeletal muscle and heart enhancer regions was used. This short $250 \mathrm{bp}$ promoter fragment increased expression in lens and decreased expression in skeletal muscle. It also appeared to increase notochord expression. The significance of $\alpha$-crystallin notochord expression is not known, although it was previously identified in mouse embryos (Gernold et al., 1993). These differences in expression produced with each mouse $\alpha B-$ crystallin promoter length are some of our best evidence that $\alpha$-crystallin regulatory elements are conserved between mouse and zebrafish.

There are some conflicting results between studies that have examined $\alpha$-crystallin expression in zebrafish embryos. A qPCR analysis by Elicker and Hutson (2007) detected $\alpha \mathrm{A}$ - and $\alpha \mathrm{Bb}$-crystallin mRNA starting at $12 \mathrm{hpf}$, with $\alpha \mathrm{A}$ increasing steadily through $5 \mathrm{dpf}$ and $\alpha \mathrm{Bb}$ increasing more slowly. They did not detect $\alpha \mathrm{Ba}$-crystallin mRNA through $5 \mathrm{dpf}$. Another study using RT-PCR also found no $\alpha$ Ba mRNA in zebrafish embryos through $78 \mathrm{hpf}$ (Mao \& Shelden, 2006). A recent report by Zou et al. (2015) found steady expression of $\alpha \mathrm{Bb}$-crystallin between $24 \mathrm{hpf}$ and $3 \mathrm{dpf}$, similar to

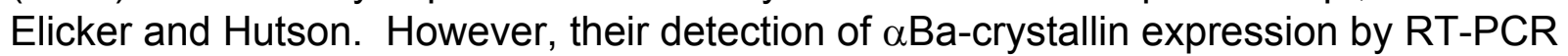
starting at $24 \mathrm{hpf}$ with steady increase through $5 \mathrm{dpf}$ differs from both past studies. Our data encompassing promoter driven GFP-expression, qPCR analysis, and proteomics help to address these discrepancies. Our detection of an increase in $\alpha A$-crystallin mRNA at $2 \mathrm{dpf}$ is consistent with the Elicker and Hutson study. This steady increase in expression was mirrored by a steady rise in $\alpha$ A-peptide detected in our embyos by 563 mass spectrometry. While our approach does not allow us to directly compare amounts 
564 of mRNA or peptide between the three zebrafish $\alpha$-crystallins, our data show that $\alpha \mathrm{A}$ 565 crystallin expression increases first, suggesting that it is the most abundant of the three $566 \alpha$-crystallins in early development. This conclusion is supported by a shotgun proteomic $\alpha \mathrm{Ba}$ - or $\alpha \mathrm{Bb}$-crystallin, in $4.5 \mathrm{dpf}$ zebrafish embryos. Our finding that $\alpha \mathrm{Ba}$-crystallin mRNA levels increased between 4 and $5 \mathrm{dpf}$ conflicts with Elicker and Hutson. Mao and Shelden only examined the $78 \mathrm{hpf}$ timepoint and may have missed the increase. Our proteomics data identified similar levels of $\alpha$ Ba-crystallin peptide between 1 and 6 $\mathrm{dpf}$, suggesting that while mRNA levels are low, and increase at $5 \mathrm{dpf}$, protein levels remain consistent during this developmental period. We did not see the large increase in aBa-crystallin mRNA identified by Zou et al. between $24 \mathrm{hpf}$ and $3 \mathrm{dpf}$. The lack of $\alpha \mathrm{Bb}$-crystallin peptide in our proteomics data is consistent with the lack of increase in mRNA from our qPCR data. We did not see the steady increase in $\alpha B b$-crystallin mRNA found by Elicker and Hutson, but rather the steady expression identified by Zou et al. However, the lack of detectable peptide, lack of increased mRNA (compared to increase in the other two zebrafish $\alpha$-crystallins), and larger variance in Ct values for technical triplicates all suggest that $\alpha \mathrm{Bb}$-crystallin is expressed at very low levels, if at all, through $5 \mathrm{dpf}$. Futhermore, the low abundance of GFP lens expression produced by both zebafish $\alpha \mathrm{B}$-crystallin promoters suggests that any significant protein expression that occurs through $5 \mathrm{dpf}$ is outside of the lens. However, our mass spectrometry did identify more $\alpha B$ a peptide in the eye compared to the rest of the zebrafish body. At some, yet unidentified, point in zebrafish development $\alpha$ Ba-crystallin expression becomes restricted to the lens.

In total the results of this study show that mammalian $\alpha$-crystallin promoter function can be screened efficiently in zebrafish embryos. Controlling regions in these promoters appear to be well conserved. Comparison of the duplicated zebrafish $\alpha \mathrm{B}$-crystallin promoters provides insight into how the function of the single mouse $\alpha B$-crystallin may have been divided between its two zebrafish orthologs. We also show that the lens specificity of zebrafish $\alpha$ Ba-crystallin seen in adults does not occur in the embryo. Variation in the temporospatial expression produced by the ten promoter fragments analyzed in this study provide a new toolset for directing the expression of introduced proteins in various embryonic zebrafish tissues at different stages of development. Our combined analysis of zebrafish $\alpha$-crystallin promoter activity, mRNA expression and protein abundance also clarifies discrepancies in the literature about when and where these genes are expressed. The ease with which engineered promoters can be injected into zebrafish embryos and their expression patterns visualized makes this model species ideal for analyses of protein expression regulation. Future studies that combine these promoter based approaches with the expanding ability to engineer the zebrafish genome via techniques such as CRISPR/Cas9 will allow the manipulation of protein expression to test hypotheses about lens crystallin function and its relation to lens biology and disease. 
608 Acknowledgements: We would like to thank Joram Piatigorsky for early conversations 609 during the development of this project and his willingness to provide insight into lens 610 crystallin promoter function. Jeff Gross served as a technical consultant on our work 611 with zebrafish, Tea Meulia provided technical help with confocal microscopy

612 (http://mcic.osu.edu/home), and Andor Kiss provided helpful feedback during the 613 drafting of this manuscript. Ashland University undergraduate student Cassie Craig 614 contributed to the characterization of zebrafish $\alpha \mathrm{B}$-crystallin promoters.

615

616 


\section{References}

618

619

620

621

622

623

624

625

626

627

628

629

630

631

632

633

634

635

636

637

638

639

640

641

642

643

644

645

646

647

648

649

650

651

652

653

654

655

656

657

658

659

660

661

662

Bourmaud A, Gallien S, Domon B 2016. Parallel reaction monitoring using quadrupoleOrbitrap mass spectrometer: Principle and applications. PROTEOMICS 16:21462159. DOI: 10.1002/pmic.201500543.

Bustin SA, Benes V, Garson JA, Hellemans J, Huggett J, Kubista M, Mueller R, Nolan T, Pfaffl MW, Shipley GL, Vandesompele J, Wittwer CT 2009. The MIQE guidelines: minimum information for publication of quantitative real-time PCR experiments. Clinical Chemistry 55:611-622. DOI: 10.1373/clinchem.2008.112797.

Chen WV, Fielding Hejtmancik J, Piatigorsky J, Duncan MK 2001. The mouse beta B1crystallin promoter: strict regulation of lens fiber cell specificity. Biochimica et biophysica acta 1519:30-38.

Chhetri J, Jacobson G, Gueven N 2014. Zebrafish—on the move towards ophthalmological research. Eye 28:367-380. DOI: 10.1038/eye.2014.19.

Clemens DM, Németh-Cahalan KL, Trinh L, Zhang T, Schilling TF, Hall JE 2013. In vivo analysis of aquaporin 0 function in zebrafish: permeability regulation is required for lens transparency. Investigative ophthalmology \& visual science 54:5136-5143. DOI: $10.1167 /$ iovs.13-12337.

Dahlman JM, Margot KL, Ding L, Horwitz J, Posner M 2005. Zebrafish alpha-crystallins: protein structure and chaperone-like activity compared to their mammalian orthologs. Molecular vision 11:88-96.

Davidson AE, Balciunas D, Mohn D, Shaffer J, Hermanson S, Sivasubbu S, Cliff MP, Hackett PB, Ekker SC 2003. Efficient gene delivery and gene expression in zebrafish using the Sleeping Beauty transposon. Developmental Biology 263:191202.

Dubin RA, Gopal-Srivastava R, Wawrousek EF, Piatigorsky J 1991. Expression of the murine alpha B-crystallin gene in lens and skeletal muscle: identification of a muscle-preferred enhancer. Molecular and cellular biology 11:4340-4349.

Elicker KS, Hutson LD 2007. Genome-wide analysis and expression profiling of the small heat shock proteins in zebrafish. Gene 403:60-69. DOI: 10.1016/j.gene.2007.08.003.

Gernold M, Knauf U, Gaestel M, Stahl J, Kloetzel PM 1993. Development and tissuespecific distribution of mouse small heat shock protein hsp25. Developmental genetics 14:103-111. DOI: 10.1002/dvg.1020140204.

Gestri G, Link BA, Neuhauss SCF 2012. The visual system of zebrafish and its use to model human ocular Diseases. Developmental neurobiology 72:302-327. DOI: 10.1002/dneu.20919.

Goishi K, Shimizu A, Najarro G, Watanabe S, Rogers R, Zon LI, Klagsbrun M 2006. AlphaA-crystallin expression prevents gamma-crystallin insolubility and cataract formation in the zebrafish cloche mutant lens. Development (Cambridge, England) 133:2585-2593. DOI: 10.1242/dev.02424.

Gopal-Srivastava R, Piatigorsky J 1994. Identification of a lens-specific regulatory region (LSR) of the murine alpha B-crystallin gene. Nucleic acids research 22:12811286.

Gopal-Srivastava R, Kays WT, Piatigorsky J 2000. Enhancer-independent promoter activity of the mouse alphaB-crystallin/small heat shock protein gene in the lens and 
663

664

665

666

667

668

669

670

671

672

673

674

675

676

677

678

679

680

681

682

683

684

685

686

687

688

689

690

691

692

693

694

695

696

697

698

699

700

701

702

703

704

705

706

707

708

cornea of transgenic mice. Mechanisms of development 92:125-134.

Greiling TMS, Clark JI 2009. Early lens development in the zebrafish: a threedimensional time-lapse analysis. Developmental dynamics : an official publication of the American Association of Anatomists 238:2254-2265. DOI: 10.1002/dvdy.21997.

Greiling TMS, Aose M, Clark JI 2010. Cell fate and differentiation of the developing ocular lens. Investigative ophthalmology \& visual science 51:1540-1546. DOI: 10.1167/iovs.09-4388.

Greiling TMS, Houck SA, Clark JI 2009. The zebrafish lens proteome during development and aging. Molecular vision 15:2313-2325.

Haynes JI, Duncan MK, Piatigorsky J 1996. Spatial and temporal activity of the alpha Bcrystallin/small heat shock protein gene promoter in transgenic mice. Developmental dynamics : an official publication of the American Association of Anatomists 207:7588. DOI: 10.1002/(SICI)1097-0177(199609)207:1<75::AID-AJA8>3.0.CO;2-T.

Hou H-H, Kuo MY-P, Luo Y-W, Chang B-E 2006. Recapitulation of human betaB1crystallin promoter activity in transgenic zebrafish. Developmental dynamics : an official publication of the American Association of Anatomists 235:435-443. DOI: 10.1002/dvdy.20652.

Hough RB, Avivi A, Davis J, Joel A, Nevo E, Piatigorsky J 2002. Adaptive evolution of small heat shock protein/alpha B-crystallin promoter activity of the blind subterranean mole rat, Spalax ehrenbergi. Proceedings of the National Academy of Sciences of the United States of America 99:8145-8150. DOI: 10.1073/pnas.122231099.

Kent WJ, Sugnet CW, Furey TS, Roskin KM, Pringle TH, Zahler AM, Haussler D 2002. The human genome browser at UCSC. Genome Research 12:996-1006. DOI: 10.1101/gr.229102.

Kleinjan DA, Bancewicz RM, Gautier P, Dahm R, Schonthaler HB, Damante G, Seawright A, Hever AM, Yeyati PL, van Heyningen V, Coutinho P 2008. Subfunctionalization of duplicated zebrafish pax6 genes by cis-regulatory divergence. PLoS genetics 4:e29. DOI: 10.1371/journal.pgen.0040029.

Koteiche HA, Claxton DP, Mishra S, Stein RA, McDonald ET, McHaourab HS 2015. Species-Specific Structural and Functional Divergence of $\alpha$-Crystallins: Zebrafish $\alpha \mathrm{Ba}-$ and Rodent $\alpha \mathrm{A}$ ins-Crystallin Encode Activated Chaperones. Biochemistry 54:5949-5958. DOI: 10.1021/acs.biochem.5b00678.

Kurita R, Sagara H, Aoki Y, Link BA, Arai K-I, Watanabe S 2003. Suppression of lens growth by alphaA-crystallin promoter-driven expression of diphtheria toxin results in disruption of retinal cell organization in zebrafish. Developmental Biology 255:113127.

MacLean B, Tomazela DM, Shulman N, Chambers M, Finney GL, Frewen B, Kern R, Tabb DL, Liebler DC, MacCoss MJ 2010. Skyline: an open source document editor for creating and analyzing targeted proteomics experiments. Bioinformatics (Oxford, England) 26:966-968. DOI: 10.1093/bioinformatics/btq054.

Mao L, Shelden EA 2006. Developmentally regulated gene expression of the small heat shock protein Hsp27 in zebrafish embryos. Gene expression patterns : GEP 6:127133. DOI: 10.1016/j.modgep.2005.07.002.

McCurley AT, Callard GV 2008. Characterization of housekeeping genes in zebrafish: male-female differences and effects of tissue type, developmental stage and 
712

713

714

715

716

717

718

719

720

721

722

723

724

725

726

727

728

729

730

731

732

733

734

735

736

737

738

739

740

741

742

743

744

745

746

747

748

749

750

751

752

753

754

chemical treatment. BMC molecular biology 9:102. DOI: 10.1186/1471-2199-9-102.

Morris AC 2011. The genetics of ocular disorders: Insights from the zebrafish. Birth defects research. Part C, Embryo today : reviews 93:215-228. DOI: 10.1002/bdrc.20211.

Posner M, Hawke M, Lacava C, Prince CJ, Bellanco NR, Corbin RW 2008. A proteome map of the zebrafish (Danio rerio) lens reveals similarities between zebrafish and mammalian crystallin expression. Molecular vision 14:806-814.

Posner M, Kantorow M, Horwitz J 1999. Cloning, sequencing and differential expression of alphaB-crystallin in the zebrafish, Danio rerio. Biochimica et biophysica acta 1447:271-277.

Posner M, Kiss AJ, Skiba J, Drossman A, Dolinska MB, Hejtmancik JF, Sergeev YV 2012. Functional Validation of Hydrophobic Adaptation to Physiological Temperature in the Small Heat Shock Protein aA-crystallin. PLoS ONE 7:e34438. DOI: $10.1371 /$ journal.pone.0034438.

R Core Team R: A Language and Environment for Statistical Computing.

Reischauer S, Stone OA, Villasenor A, Chi N, Jin S-W, Martin M, Lee MT, Fukuda N, Marass M, Witty A, Fiddes I, Kuo T, Chung W-S, Salek S, Lerrigo R, Alsiö J, Luo S, Tworus D, Augustine SM, Mucenieks S, Nystedt B, Giraldez AJ, Schroth GP, Andersson O, Stainier DYR 2016. Cloche is a bHLH-PAS transcription factor that drives haemato-vascular specification. Nature 535:294-298. DOI: doi:10.1038/nature18614.

RStudio Team RStudio: Integrated Development Environment for R.

Runkle S, Hill J, Kantorow M, Horwitz J, Posner M 2002. Sequence and spatial expression of zebrafish (Danio rerio) alphaA-crystallin. Molecular vision 8:45-50.

Smith AA, Wyatt K, Vacha J, Vihtelic TS, Zigler JS, Wistow GJ, Posner M 2006. Gene duplication and separation of functions in alphaB-crystallin from zebrafish (Danio rerio). The FEBS journal 273:481-490. DOI: 10.1111/j.1742-4658.2005.05080.x.

Swamynathan SK, Piatigorsky J 2002. Orientation-dependent influence of an intergenic enhancer on the promoter activity of the divergently transcribed mouse Shsp/alpha B-crystallin and Mkbp/HspB2 genes. The Journal of biological chemistry 277:49700-49706. DOI: 10.1074/jbc.M209700200.

Tang R, Dodd A, Lai D, McNabb WC, Love DR 2007. Validation of zebrafish (Danio rerio) reference genes for quantitative real-time RT-PCR normalization. Acta Biochimica et Biophysica Sinica 39:384-390.

Van de Peer Y, Taylor JS, Meyer A 2003. Are all fishes ancient polyploids? Journal of structural and functional genomics 3:65-73.

Vihtelic TS 2008. Teleost lens development and degeneration. International review of cell and molecular biology 269:341-373. DOI: 10.1016/S1937-6448(08)01006-X.

Wages P, Horwitz J, Ding L, Corbin RW, Posner M 2013. Changes in zebrafish (Danio rerio) lens crystallin content during development. Molecular vision 19:408-417.

Wistow GJ, Piatigorsky J 1988. Lens Crystallins: The Evolution and Expression of Proteins for a Highly Specialized Tissue. dx.doi.org 57:479-504. DOI:

10.1146/annurev.bi.57.070188.002403.

Wistow G, Wyatt K, David L, Gao C, Bateman O, Bernstein S, Tomarev S, Segovia L, Slingsby C, Vihtelic T 2005. gammaN-crystallin and the evolution of the betagamma-crystallin superfamily in vertebrates. The FEBS journal 272:2276-2291. 
DOI: 10.1111/j.1742-4658.2005.04655.x.

Wolf L, Yang Y, Wawrousek E, Cvekl A 2008. Transcriptional regulation of mouse alpha A-crystallin gene in a $148 \mathrm{~kb}$ Cryaa BAC and its derivates. BMC developmental biology 8:88. DOI: 10.1186/1471-213X-8-88.

Xie Q, McGreal R, Harris R, Gao CY, Liu W, Reneker LW, Musil LS, Cvekl A 2016. Regulation of c-Maf and $\alpha \mathrm{A}-\mathrm{Crystallin}$ in Ocular Lens by Fibroblast Growth Factor Signaling. Journal of Biological Chemistry 291:3947-3958. DOI: 10.1074/jbc.M115.705103.

Yang Y, Cvekl A 2005. Tissue-specific Regulation of the Mouse aA-crystallin Gene in Lens via Recruitment of Pax6 and c-Maf to its Promoter. Journal of molecular biology 351:453-469. DOI: 10.1016/j.jmb.2005.05.072.

Yang Y, Chauhan BK, Cveklova K, Cvekl A 2004. Transcriptional regulation of mouse alphaB- and gammaF-crystallin genes in lens: opposite promoter-specific interactions between Pax6 and large Maf transcription factors. Journal of molecular biology 344:351-368. DOI: 10.1016/j.jmb.2004.07.102.

Yang Y, Stopka T, Golestaneh N, Wang Y, Wu K, Li A, Chauhan BK, Gao CY, Cveklova K, Duncan MK, Pestell RG, Chepelinsky AB, Skoultchi Al, Cvekl A 2006. Regulation of alphaA-crystallin via Pax6, c-Maf, CREB and a broad domain of lens-specific chromatin. The EMBO journal 25:2107-2118. DOI: 10.1038/sj.emboj.7601114.

Zou P, Wu S-Y, Koteiche HA, Mishra S, Levic DS, Knapik E, Chen W, McHaourab HS 2015. A conserved role of $\alpha A$-crystallin in the development of the zebrafish embryonic lens. Experimental Eye Research 138:104-113. DOI: 10.1016/j.exer.2015.07.001. 


\section{Figure 1 (on next page)}

Confocal imagery showing representative sites of GFP expression produced by mouse and zebrafish $\alpha$-crystallin promoters

Examples of lens expression produced with a zebrafish $\alpha \mathrm{A}$ promoter ( $\mathrm{A}$ and $\mathrm{B}$ ). Various sites of extraocular expression shown as single z-planes (on left) and as 3-dimensional renders (on right) for skeletal muscle produced with a mouse $\alpha B$ promoter ( $C$ and $D$ ); for notochord produced with a zebrafish $\alpha \mathrm{Bb}$ promoter ( $\mathrm{E}$ and $\mathrm{F}$ ); dorsal to the yolk produced with a zebrafish aA promoter ( $\mathrm{G}$ and $\mathrm{H}$ ). 

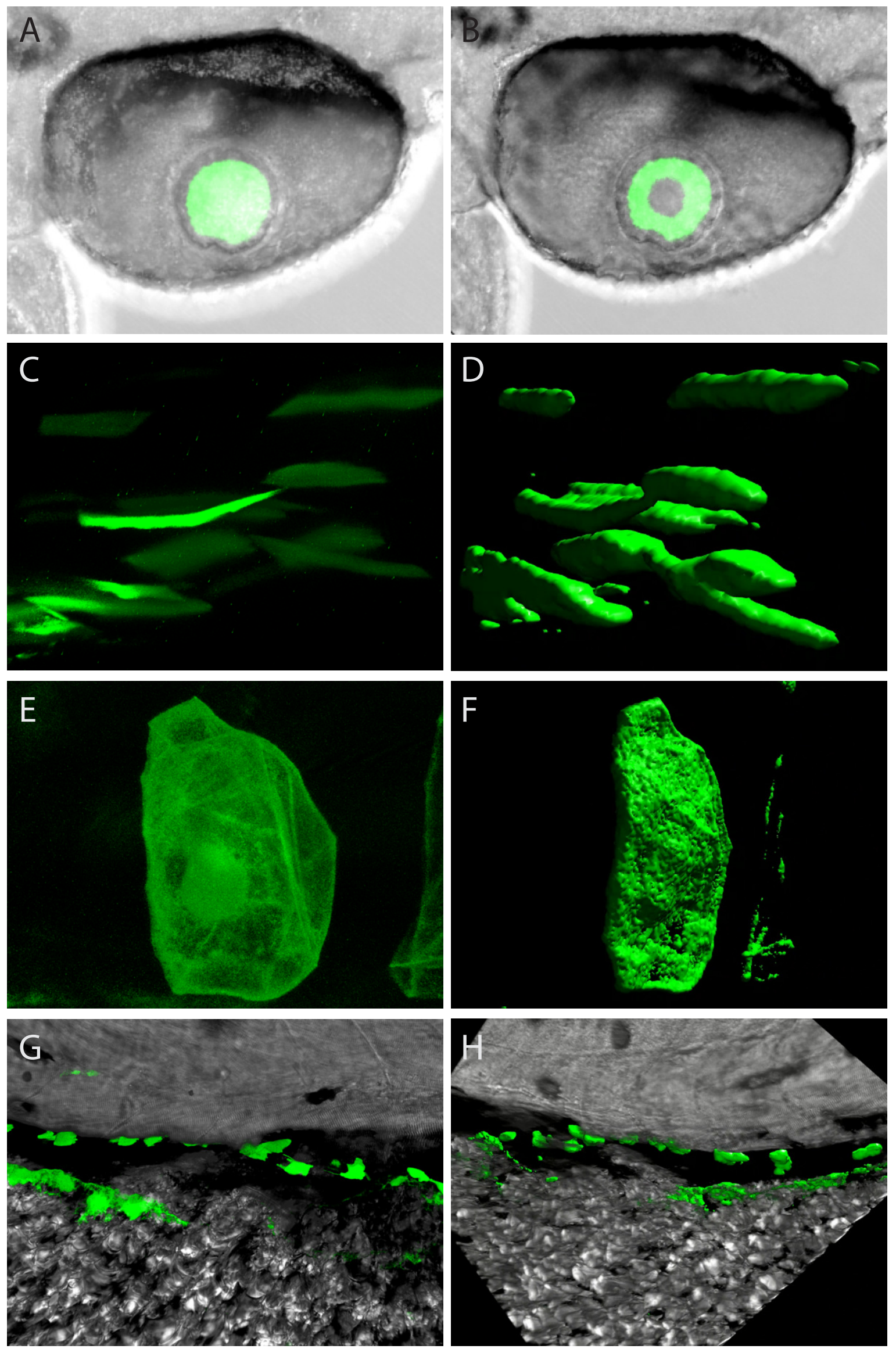


\section{Figure 2 (on next page)}

Comparison of mouse and zebrafish $\alpha$ A-crystallin chromosomal arrangement and their ability to drive GFP expression in zebrafish embryos

The structural and functional conservation of mammalian and zebrafish $\alpha \mathrm{A}$-crystallin is mirrored in their shared syntenic relationship with hsf2bp (A). Vertical bars note exons, thin horizontal lines note introns and arrows show direction of transcription. The promoter regions for each gene produced similar temporal and spatial expression patterns (B-E), with expression almost exclusively restricted to the lens. The extent of lens expression varied for both orthologous promoters (compare $B$ to $C$ for mouse and $D$ to $E$ for zebrafish). 


\section{A Zebrafish chromosome 1}

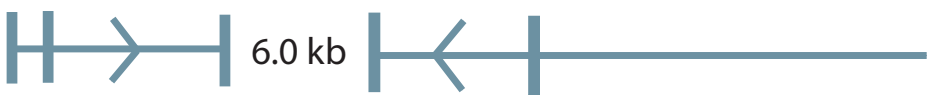

$\alpha$ A-crystallin

hsf2bp

\section{Mouse chromosome 17}

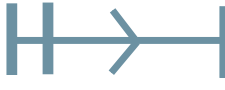

$\alpha A$-crystallin $162.5 \mathrm{~kb}$

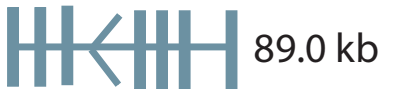

Sik1

hsf2bp

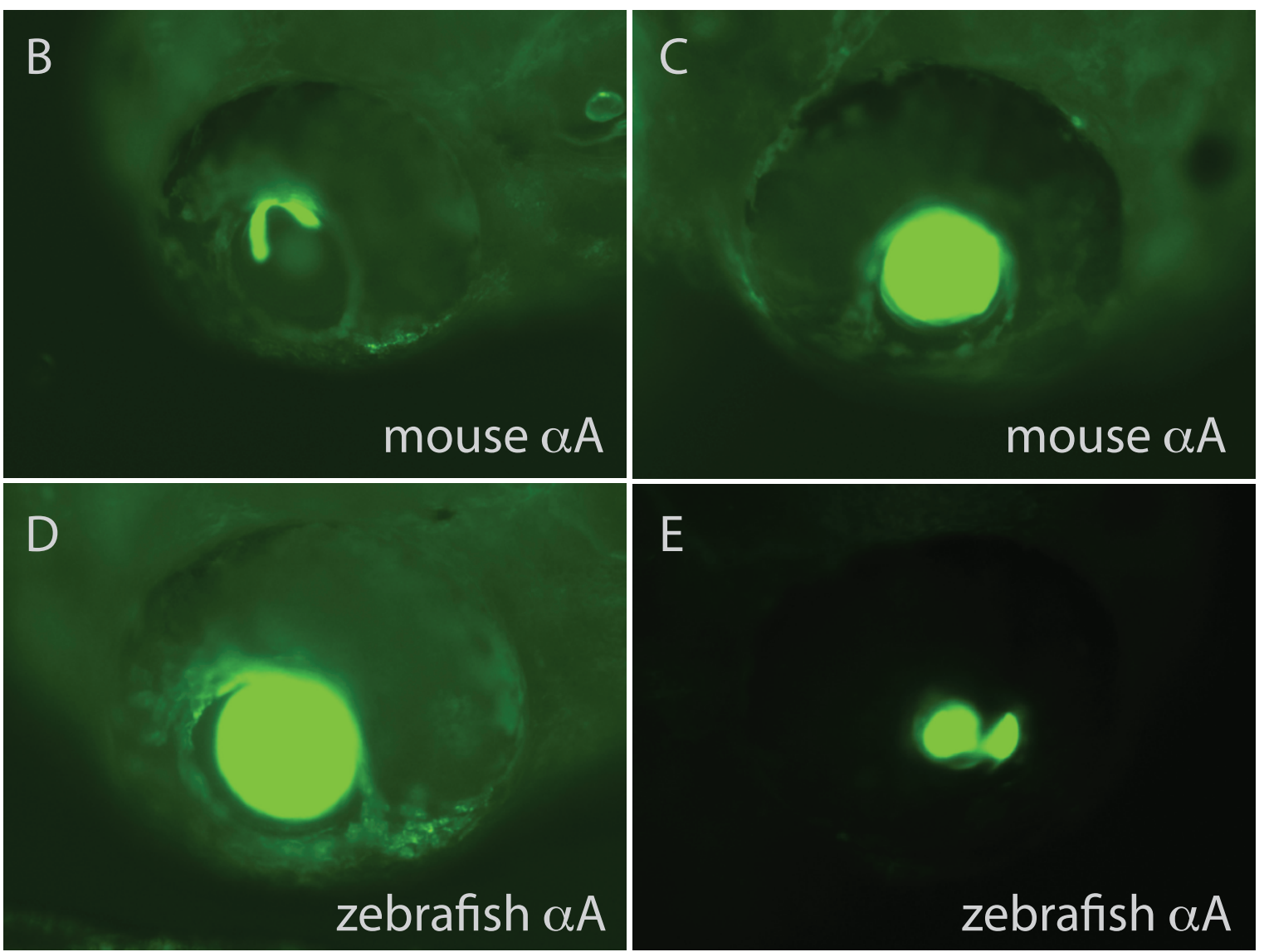




\section{Figure 3 (on next page)}

Mouse $\alpha \mathrm{B}$-crystallin promoter fragments produced native expression in zebrafish embryos

Enhancer elements of a promoter upstream of mouse $\alpha \mathrm{B}$-crystallin were previously shown to regulate expression in skeletal muscle (sm), heart and lens (Isr1 and 2) ( $\mathrm{A}$; adapted from (Swamynathan \& Piatigorsky, 2002) ). Fragments containing 250 bp, 0.8 and 1.5 kb lengths of this promoter produced GFP expression in zebrafish embryo notochord (B-D), skeletal muscle (E), lens (F) and heart (G; arrows). Panel E shows GFP expression in both slow (noted by *) and fast twitch (noted by arrows) muscle fibers. The yolk remaining in these embryos is autofluorescent. The $250 \mathrm{bp}$ fragment, which lacked the heart and skeletal muscle enhancer, produced less frequent GFP expression in these tissues, and GFP expression onset was slightly earlier (Tables 3 and 4). 
A

Mouse chromosome 9

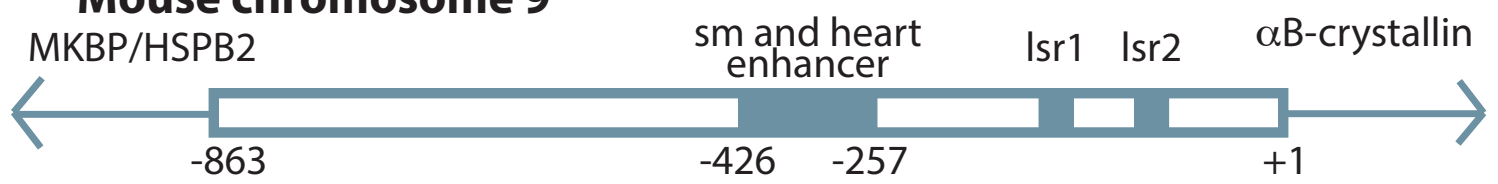

B
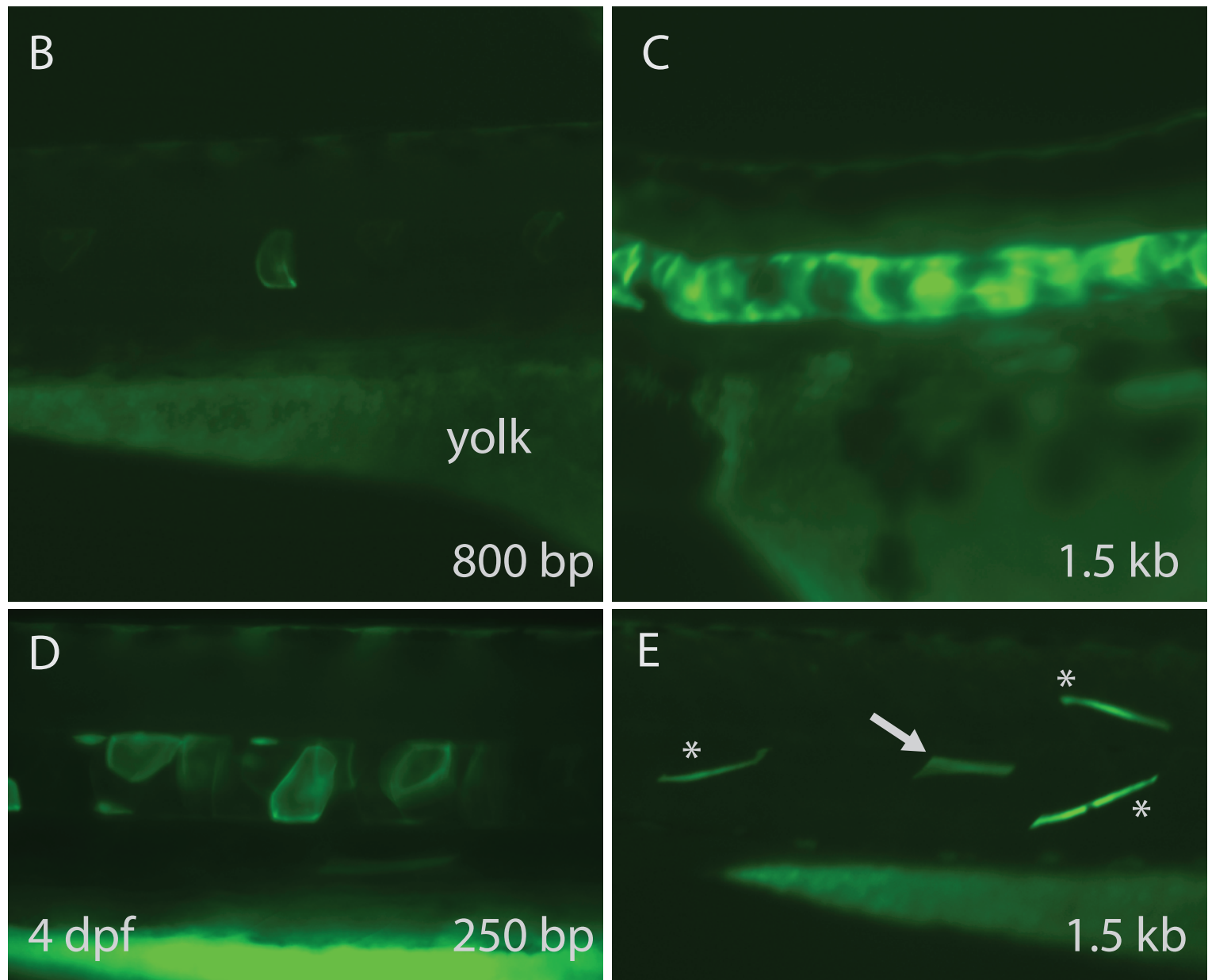

F

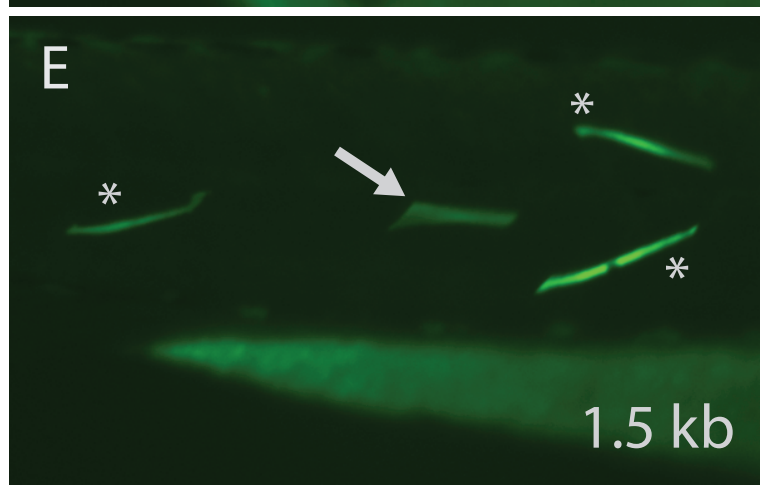

G

800 bp

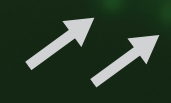

$800 \mathrm{bp}$ 


\section{Figure 4}

The paralogous zebrafish $\alpha \mathrm{Ba}$ - and $\alpha \mathrm{Bb}$-crystallin promoters produced similar, but distinct, GFP expression profiles

Zebrafish $\alpha \mathrm{Bb}$-crystallin has the same syntenic relationship with $\mathrm{Hspb2}$ as mouse $\alpha \mathrm{B}$ crystallin, although the intergenic region between the two genes is much larger in the zebrafish (A). The zebrafish $\alpha$ Ba-crystallin paralog has moved to a separate chromosome. Both zebrafish paralogs produced GFP expression most often in notochord (B) and skeletal muscle (C). The $\alpha \mathrm{Ba}$ paralog drove expression in these tissues equally while $\alpha \mathrm{Bb}$ was more active in skeletal muscle (D). Expression in lens (E) and extralenticular regions of the eye was more rare. Images shown are representative with the details of GFP expression not differing noticeably between paralogs or the promoter length used. 
A

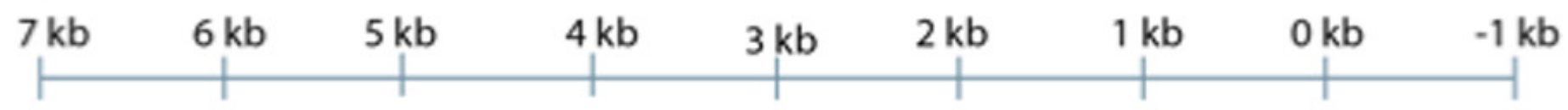

\section{Zebrafish chromosome 5}

Hspb2

\section{Mouse chromosome 9}

$\alpha$ Bb-crystallin

\section{Zebrafish chromosome 15}

\section{Hspb2}

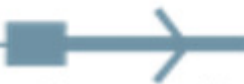

$\alpha B$-crystallin
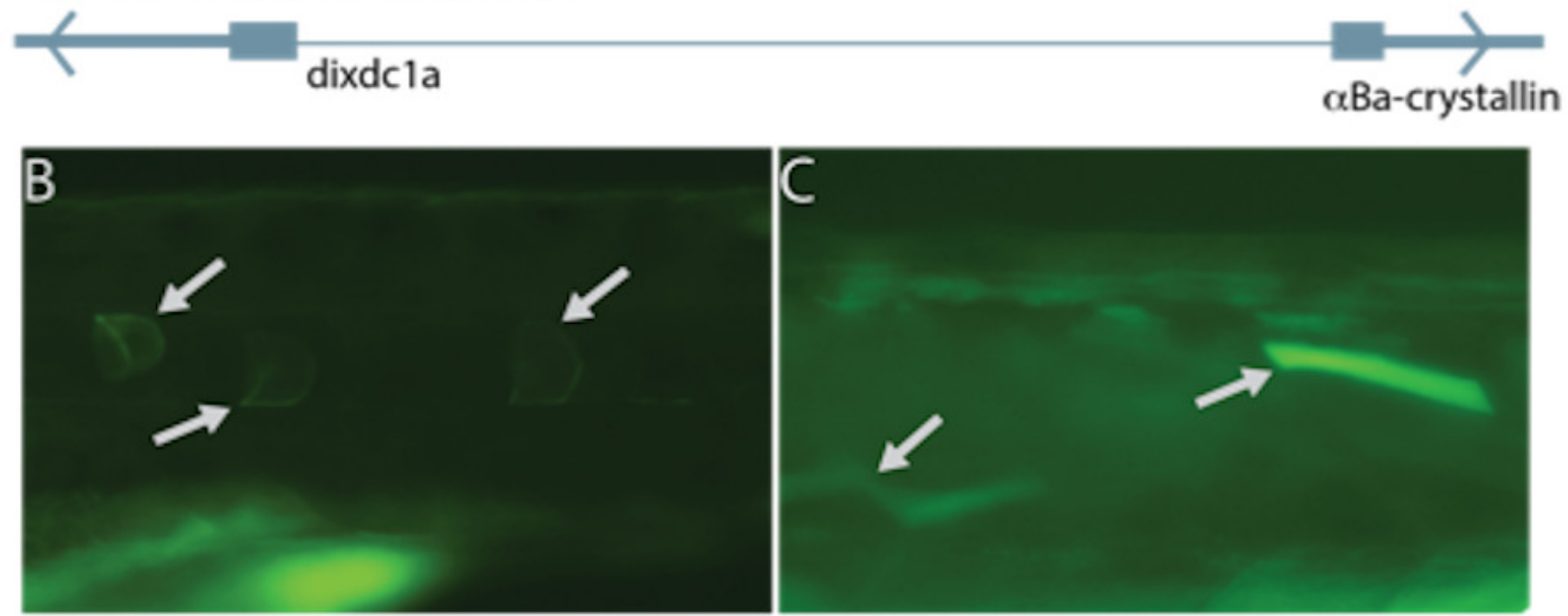

$\mathrm{D}_{100}$

E

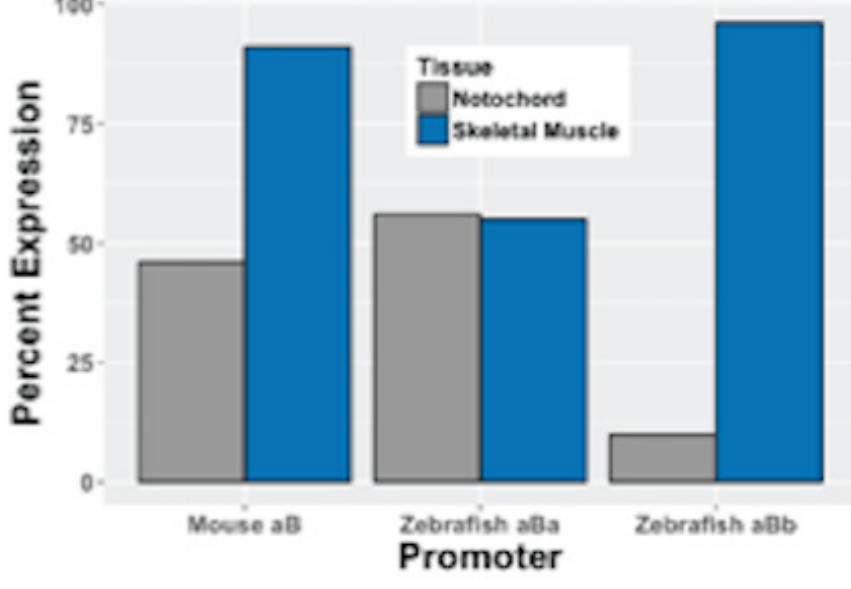




\section{Figure 5}

qPCR analysis of $\alpha$-crystallin expression in zebrafish embryos

Box and whisker plot shows delta Ct for the three zebrafish $\alpha$-crystallins, indicating mRNA levels relative to three endogenous controls from 12 hours post fertilization ( $0.5 \mathrm{dpf})$ to $5 \mathrm{dpf}$. Lower numerical $\mathrm{Ct}$ values on these inverted y-axes indicate increased expression. All three graphs show low initial baseline expression that increases in $\alpha A(A)$ and $\alpha B a-c r y s t a l l i n(B)$, but stays consistently low in $\alpha \mathrm{Bb}$-crystallin (C). Alpha A-crystallin expression increased earlier than $\alpha$ Ba-crystallin expression. Asterisks indicate statistically significant differences in expression compared to the $0.5 \mathrm{dpf}$ timepoint $(\mathrm{p}<0.05$; ANOVA with a post-hoc Tukey's HSD test). Each box plot reflects three separate biological replicates for each timepoint. The calculated Ct values for each of three technical triplicates making up each biological replicate showed variation of more than 0.5 only for the lower expressed baseline samples. Timepoints with statistically significant increased expression produced technical triplicates with Ct values within 0.5 of each other (with one exception out of 9 measurements). Three and 4-dpf samples were not analyzed for $\alpha \mathrm{A}$ - and $\alpha \mathrm{Ba}$-crystallin since no change in expression was seen for each gene between 2 and $5 \mathrm{dpf}$. 


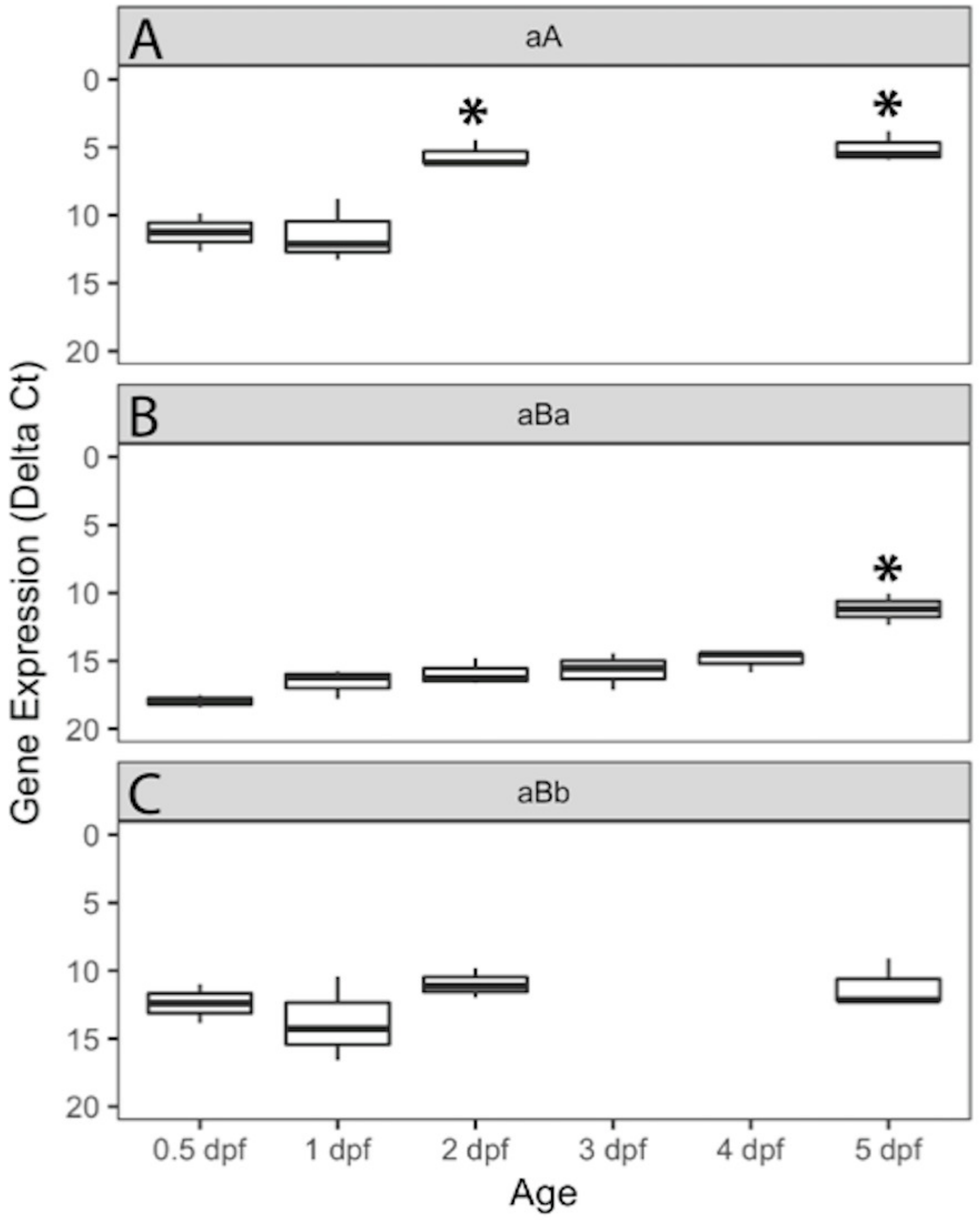




\section{Figure 6}

Relative abundance of $\alpha \mathrm{A}$ - and $\alpha \mathrm{Ba}$-crystallin proteins in zebrafish embryos during development measured by mass spectrometric parallel reaction monitoring of tryptic peptides

(A) Changes in aA-crystallin relative abundance in whole embryos from 1-6 days post fertilization (dpf) by measurement of peak areas for the top 3 fragment ions of peptide 52-65 (NILDSSNSGVSEVR). Orange $=y 12$, blue $=y 11$, and green $=y 10$ fragment ions. The bar labeled library shows the relative proportion of these fragment ions for this peptide identified in a digest from an adult zebrafish lens, while the dotp value above each bar is a measurement of how well the observed fragment ions for this peptide in each embryo digest matched those for this peptide in a spectral library created from an adult lens digest. Note that the relative peak area for the library peptide was arbitrary set to the same value as the largest peak area for ease of comparison. (B) Relative abundance of $\alpha A$-crystallin in dissected eyes and remaining trunks of either 4 or $7 \mathrm{dpf}$ embryos. The same $\alpha \mathrm{A}$ peptide and fragment ions as measured above in A were used. (C) Measurement of $\alpha$ Ba-crystallin in whole embryos from 1-6 dpf by measurement of peak areas for the top 3 fragment ions of peptide 79-88 (HFSPDELTVK). Orange $=b 2$, blue $=y 9$, and green $=y 8$ fragment ions. (D) Relative abundance of $\alpha \mathrm{Ba}$-crystallin in dissected eyes and remaining trunks of either 4 or $7 \mathrm{dpf}$ embryos. The same $\alpha B$ a peptide and fragment ions as measured in $\mathrm{C}$ were used. Extracted ions chromatograms for the fragment ions of these peptides are shown in Supplementary Figure 2. 

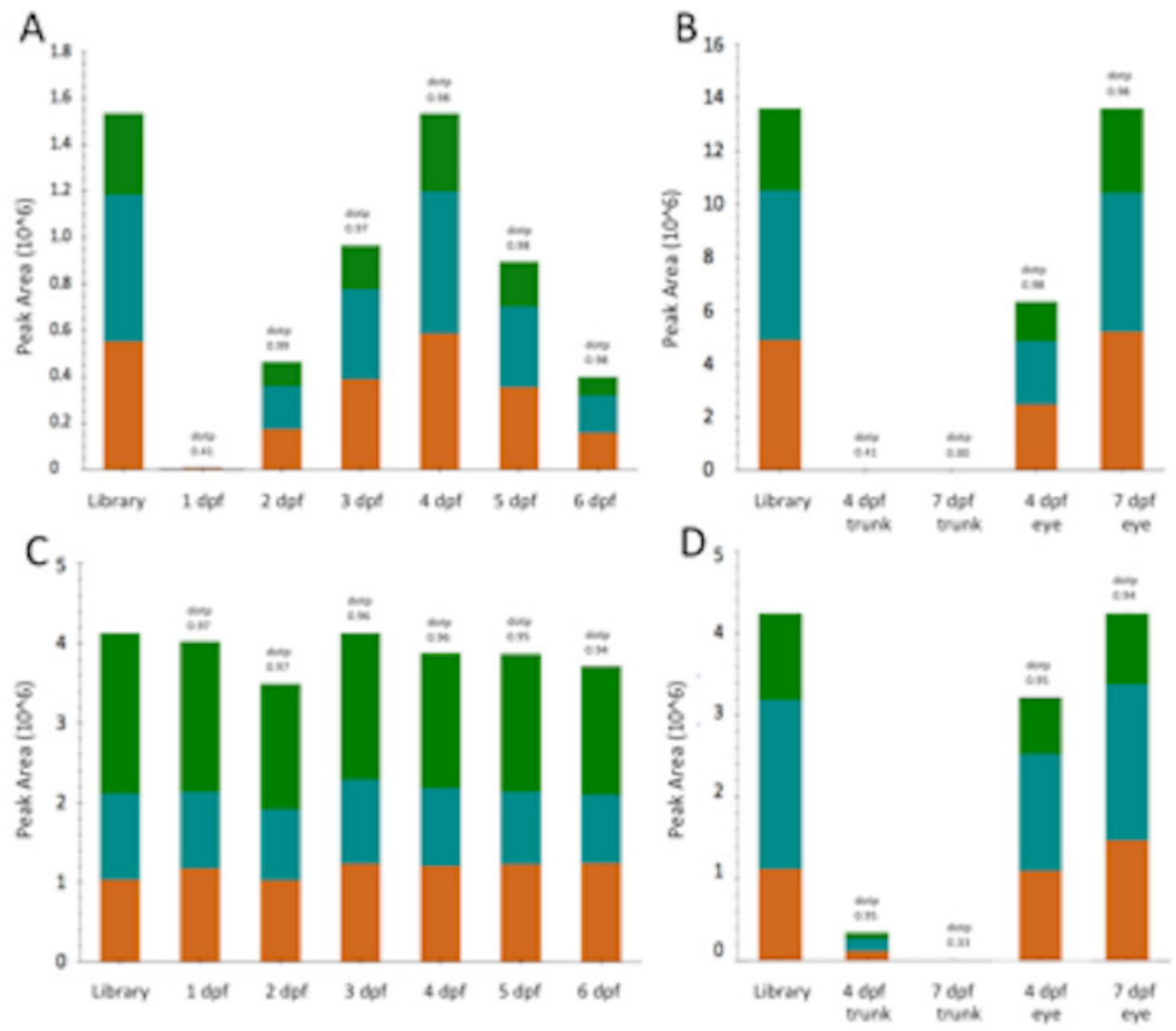


\section{Table $\mathbf{1}$ (on next page)}

Primers used to construct promoter fragments

Gene accession numbers are given for each gene as well as coordinates showing region of each promoter fragment relative to the gene's start codon. Lower case letters indicate added nucleotides for insertion into cloning plasmid. Promoter fragments were constructed through either traditional cloning methods using restriction enzymes Xhol and BamH1 or by Gibson assembly. 
1 Table 1. Primers used to construct promoter fragments. Gene accession numbers are given for each gene as well as 2 coordinates showing region of each promoter fragment relative to the gene's start codon. Lower case letters indicate added 3 nucleotides for insertion into cloning plasmid. Promoter fragments were constructed through either traditional cloning 4 methods using restriction enzymes Xho1 and BamH1 or by Gibson assembly. The mouse $\alpha$ A-crystallin promoter was provided 5 by the laboratory of Dr. Ales Cvekl.

6

7

\begin{tabular}{|c|c|c|c|c|c|}
\hline Gene & $\begin{array}{l}\text { Gene } \\
\text { Accession \# }\end{array}$ & Coordinates & Forward Primer & Reverse Primer & $\begin{array}{c}\text { Construction } \\
\text { Method }\end{array}$ \\
\hline \multicolumn{6}{|c|}{ 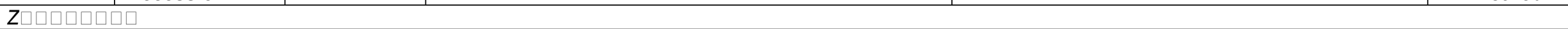 } \\
\hline$\alpha \mathrm{A} \mathrm{1kb}$ & NM_152950.2 & $-1028 /-1$ & ggaattctcgagTGGAGACCCCTGATTAATA & ggaattggatccAATGTCAGACCTGGTAACT & Xho1/BamH1 \\
\hline$\alpha \mathrm{Ba} 3 \mathrm{~kb}$ & NM_131157.1 & $-3000 /-1$ & $\begin{array}{l}\text { ctaccggactcagatcGAAAAAAAAAAAAGAAAGAAAGAAA } \\
\text { AGAAAG }\end{array}$ & ccatggtggcgaccggtgTGTACCTTAGTTTGGAGC & Gibson \\
\hline$\alpha \mathrm{Bb} 1 \mathrm{~kb}$ & \multirow{4}{*}{$\begin{array}{l}\text { NM_001002670 } \\
.2\end{array}$} & $-1074 /-1$ & ggaattctcgagTTCAATGGTGCGCTGT & ggaattggatccTTTGAGTCTGGGCCTCTT & Xho1/BamH1 \\
\hline$\alpha \mathrm{Bb} 2 \mathrm{~kb}$ & & $-2092 /-1$ & ggaattctcgagAGACGTTACAGTGGGCTA & ggaattggatccTTTGAGTCTGGGCCTCTT & Xho1/BamH1 \\
\hline$\alpha \mathrm{Bb} 4 \mathrm{~kb}$ & & $-3999 /-1$ & ctaccggactcagatcCGCACCGTACAAAGATTTG & ccatggtggcgaccggtgTTTGAGTCTGGGCCTCTTC & Gibson \\
\hline$\alpha \mathrm{Bb} 5 \mathrm{~kb}$ & & $-4999 /-1$ & ctaccggactcagatcAATTTAGACCTGCTTTTAGTTGG & ccatggtggcgaccggtgTTTGAGTCTGGGCCTCTTC & Gibson \\
\hline \multicolumn{6}{|l|}{ Mouse } \\
\hline$\alpha \mathrm{A}$ & NM_001278570 & \multicolumn{3}{|c|}{$-7706 /-7492$ and $-1800 /+46$} & \\
\hline$\alpha \mathrm{B} 0.25 \mathrm{~kb}$ & \multirow[t]{3}{*}{ CT010341 } & $-259 /-1$ & cgagctcaagcttcgGTGAAACAAGACCATGAC & ccatggtggcgaccggtgTGTGGCTAGATGAATGCAG & Gibson \\
\hline$\alpha \mathrm{B} 0.8 \mathrm{~kb}$ & & $-833 /-1$ & ggaattctcgagGTGCAGCTATGAGGGTGTGA & ggaattggatccTGTGGCTAGATGAATGCAGA & Xho1/BamH1 \\
\hline$\alpha \mathrm{B} 1.5 \mathrm{~kb}$ & & $-1501 /-1$ & ggaattctcgagAAAGCAAGAGGCAGGATGAG & ggaattggatccTGTGGCTAGATGAATGCAGA & Xho1/BamH1 \\
\hline
\end{tabular}

8

9 


\section{Table 2 (on next page)}

Primers used for qPCR analysis of zebrafish $\alpha$-crystallin expression

Primer efficiency and $R^{2}$ values were calculated using a standard curve procedure described in the Materials and Methods section. 
1 Table 2. Primers used for qPCR analysis of zebrafish alpha crystallin expression. All primers have been used in previous 2 publications (see Methods for references). Standard curve qPCR reactions were used to calculate the efficiency and R2 value 3 for each primer pair when amplifying cDNA from transcribed adult zebrafish lens RNA.

4

5

\begin{tabular}{|l|l|c|c|c|c|}
\hline Gene & Primer Sequence & $\begin{array}{c}\text { Product Size } \\
(\mathbf{b p})\end{array}$ & Accession \# & Efficiency $^{\mathbf{R}^{\mathbf{2}}}$ \\
\hline$\alpha$ A-crystallin & $\begin{array}{l}\text { F: 5'ATGGCCTGCTCACTCTTTGT3' } \\
\text { R: 5'CCCACTCACACCTCCATACC3' }\end{array}$ & 159 & AY035778 & 84.3 & 0.965 \\
\hline$\alpha$ Ba-crystallin & $\begin{array}{l}\text { F: 5'CCCAGGCTTCTTCCCTTATC3' } \\
\text { R: 5'GTGCTTCACATCCAGGTTGA3' }\end{array}$ & 196 & NM_131157 & 97.5 & 0.994 \\
\hline$\alpha$ Bb-crystallin & $\begin{array}{l}\text { F: 5'CCTATCGACGGCAAATGTT3' } \\
\text { R: 5'GGCATCAGCAGCAGACAATA3' }\end{array}$ & 128 & NM_001002670 & 93.8 & 0.995 \\
\hline EF-1 $\alpha$ & $\begin{array}{l}\text { F: 5'CAGCTGATCGTTGGAGTCAA3' } \\
\text { R: 5'TGTATGCGCTGACTTCCTTG3' }\end{array}$ & 94 & AY422992 & 85.4 & 0.999 \\
\hline$\beta$-actin & $\begin{array}{l}\text { F: 5'CGAGCAGGAGATGGGAACC3' } \\
\text { R: 5'CAACGGAACGCTCATTGC3' }\end{array}$ & 102 & FJ915059 & 80.5 & 0.997 \\
\hline Rpl13A & $\begin{array}{l}\text { F: 5'TCTGGAGGACTGTAAGAGGTATGC3' } \\
\text { R: 5'AGACGCACAATCTTGAGAGCAG3' }\end{array}$ & 148 & NM_212784 & 96.1 & 0.999 \\
\hline
\end{tabular}

6

7

8 


\section{Table 3(on next page)}

Location of promoter activity

Total embryos shows the number of separate embryos examined after injection with each indicated promoter fragment. Percentage of embryos shows the proportion of GFP-expressing embryos with observable GFP in each tissue. A "O" indicates no embryos expressed GFP in that tissue. 
1 Table 3. Location of Promoter Activity. Total embryos shows the number of separate 2 embryos examined after injection with each indicated promoter fragment. Percentage of 3 embryos shows the proportion of GFP-expressing embryos with observable GFP in each 4 tissue. A "O" indicates no embryos expressed GFP in that tissue.

5

6 Zebrafish Total Percentage of embryos

7 Promoters Embryos Lens Eye NC $\quad$ SM Heart

$8 \quad 1 \mathrm{~kb} \alpha \mathrm{A} \quad 62$

9

10

11

12

13

14

15

16

17

18

19

20

21

22

23

24

$\begin{array}{ccccccc}3 \mathrm{~kb} \alpha \mathrm{Ba} & 90 & 3 & 7 & 56 & 55 & 0 \\ & & & & & & \\ 1 \mathrm{~kb} \alpha \mathrm{Bb} & 67 & 0 & 5 & 5 & 90 & 0 \\ 2 \mathrm{~kb} \alpha \mathrm{Bb} & 59 & 0 & 6 & 3 & 92 & 3 \\ 4 \mathrm{~kb} \alpha \mathrm{Bb} & 51 & 0 & 0 & 20 & 100 & 0 \\ 5 \mathrm{~kb} \alpha \mathrm{Bb} & 90 & 4 & 4 & 7 & 97 & 0\end{array}$

Mouse Total Percentage of embryos

\begin{tabular}{ccccccc} 
Promoters & Embryos & Lens & Eye & NC & SM & Heart \\
\hline$\alpha \mathrm{A}$ & 55 & 70 & 0 & 4 & 30 & 0
\end{tabular}

$\begin{array}{lllllll}0.25 \mathrm{~kb} \alpha \mathrm{B} & 64 & 27 & 2 & 62 & 79 & 2\end{array}$

$\begin{array}{lllllll}0.8 \mathrm{~kb} \alpha \mathrm{B} & 50 & 13 & 15 & 33 & 94 & 6\end{array}$

$\begin{array}{lllllll}1.5 \mathrm{~kb} \alpha \mathrm{B} & 67 & 15 & 5 & 42 & 100 & 12\end{array}$




\section{Table 4 (on next page)}

Timeline of promoter activity

Numbers indicate percentage of injected embryos expressing GFP in any tissue at indicated timepoints. Lack of expression is noted with an " $\mathrm{O}$ " and "--" indicates that no embryos were observed at that timepoint. 
1 Table 4. Timeline of Promoter Activity. Numbers indicate percentage of injected

2 embryos expressing GFP in any tissue at indicated timepoints. Lack of expression is noted

3 with an " 0 " and "--" indicates that no embryos were observed at that timepoint.

4

5

6

7

8

9

10

11

12

13

14

15

16

17

18

19

20

21

22

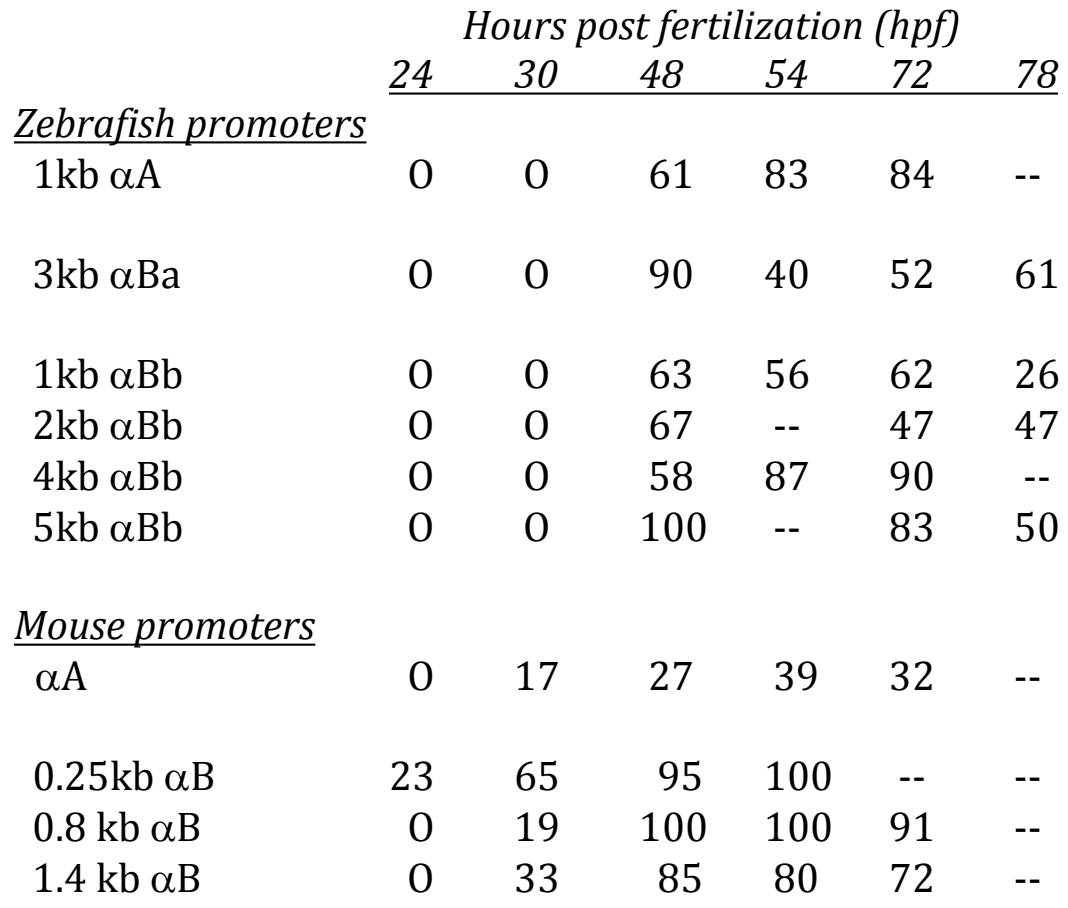

